\title{
Attosecond Spectroscopy
}

\author{
Hans Jakob Wörner ${ }^{1,2}$ and Paul B. Corkum ${ }^{2}$ \\ ${ }^{1}$ Laboratorium für Physikalische Chemie, ETH Zürich, Zürich, Switzerland \\ ${ }^{2}$ Joint Laboratory for Attosecond Science, National Research Council of Canada and University of Ottawa, Ottawa, Ontario, \\ Canada
}

\section{INTRODUCTION}

Classical spectroscopy measures the light absorbed or emitted by atoms, molecules, or condensed media. This method was powerful enough to drive the development of quantum mechanics and to determine the structure of most small molecules (Herzberg 1989, 1991a,b Huber and Herzberg 1979). Absorption and emission remained the basic tools of spectroscopy until the advent of the laser. The laser has given access to much more precise measurements, exploiting the narrow bandwidth and the coherence of the new light source. The availability of much higher intensities made nonlinear spectroscopy accessible, and over the past 50 years, nonlinear spectroscopy has added a very important new dimension to the field (Mukamel 1999, Zewail 1994). Now spectroscopy allows some of the most precise measurements in science (Udem et al. 1997, Fischer et al. 2004). The control over matter that arises from nonlinear laser spectroscopy allows the coldest matter to be produced (Chu 2002).

Attosecond science is the extension of nonlinear optics beyond the limit where perturbation theory is valid (Corkum 1993, Lewenstein et al. 1994) and new forms of spectroscopy grow from this extension. We deal with intense laser pulses. It is the extreme nonlinearity that allows the laser period to be subdivided. The current short-pulse record of 80 as (Goulielmakis et al. 2008) is less than 1/30th of the optical period of the $800-\mathrm{nm}$ light that produced it. From basic mathematics, it is clear that the carrier frequency of an 80 -as pulse is well beyond the optical range - at least 30 times the laser frequency of the 800-nm light, which

Handbook of High-resolution Spectroscopy. Edited by Martin Quack and Frédéric Merkt. (C) 2011 John Wiley \& Sons, Ltd. ISBN: 978-0-470-74959-3. means that the spectrum of the pulse must be centered above $50 \mathrm{eV}$.

Our intuition from perturbation theory is no longer useful for such a highly nonlinear process. At least 30 photons must be involved (in fact many more). With such a highorder process, we can no longer assume that higher order terms are not as important (or even more important) as the lowest order (e.g., 30 photon) term. While perturbation theory is no longer useful, classical ideas can serve as a new foundation for our intuition (Corkum 1993). Classically, electrons respond to forces - the force of the laser field. This gives rise to the term strong-field physics that is often used in place of high-intensity physics.

Although we often use classical language to describe strong-field processes, strong-field physics is not just classical physics performed on an atomic or molecular scale. It is almost impossible for an electron to lose its coherence in a fraction of a laser period, at least in the gas phase. Therefore, classical ideas can be directly mapped onto quantum mechanics (Lewenstein et al. 1994), allowing one to develop a more nuanced intuition.

Now let us look at the physics that underlies attosecond science. To produce a $50-\mathrm{eV}$ photon, a $50-\mathrm{eV}$ electron is needed. This is above the ionization potential of any neutral atom: we must be dealing with a continuum electron. Immediately we see that attosecond spectroscopy will be closely related to photoelectron spectroscopy.

The physics of attosecond pulse generation is very simple. Often it is broken into three steps (Corkum 1993). In the first step, an electron wave packet tunnels from an atom or a molecule to the continuum. Tunneling is one of the most important and fundamental processes in quantum mechanics (Hund 1927, Gamow 1929). Tunneling spectroscopy will be one of the spectroscopic methods 
that will be greatly extended by attosecond science. The discussion of the subsequent steps is followed by a description of how tunneling electrons can be observed.

Tunneling splits the total electronic wave function into two components. One component is the bound-state wave function and the other is the continuum wave packet. The field strength needed to produce a wave packet of significant amplitude each half-period is $\sim 1 / 10$ of the atomic field or $\sim 3 \times 10^{14} \mathrm{~W} \mathrm{~cm}^{-2}\left(E_{0} \sim 4 \mathrm{~V}^{-1}\right)$. Such a strong oscillating field causes the electron to oscillate. First, the electron moves away from the ion but after the field reverses, it is driven back. This is the second step. It is here that classical physics is a very good guide to our intuition.

Parts of the continuum wave packet recollide-some parts with high energy. The third step, which leads to the attosecond optical pulses (from which attosecond science gets its name), arises when the electron recombines to the original state, just photoelectron spectroscopy in reverse. In other words, attosecond extreme-ultraviolet (XUV) pulses are produced from preexisting photoelectrons: these are created by tunneling and controlled by the fundamental laser field in a classical-like manner, and their coherence with the original orbital from which they separated is retained. Thus, photoelectron spectroscopy is the second field of spectroscopy that is enhanced by attosecond technology. It gains a practical (coherent) inverse process. Photoelectron information is read in photons that do not suffer from space charge limits.

Classical photoelectron spectroscopy has been used to characterize the orbital structure of molecules (Kimura et al. 1981). This information drove the development of molecular orbital theory and quantum chemistry for many decades. The high spatial resolution achieved by the recolliding electron wave packet now permits the imaging of orbitals in a tomographic procedure (Itatani et al. 2004), thereby adding a new dimension to photoelectron spectroscopy. The attosecond resolution inherent to the method may allow the observation of ultrafast electronic rearrangements.

Now we turn to another new branch of photoelectron spectroscopy that naturally arises. Not all of the electrons that recollide with their parent ion recombine. Some of the recollision electrons will elastically scatter from their parent ion. Their de Broglie wavelength can be in the range of $\lambda=0.5-5 \AA$, nicely matching the important distances in molecules. In other words, they will diffract from their parent molecule (Spanner et al. 2004, Meckel et al. 2008). Thus, through recollision, photoelectron spectroscopy is unified with electron diffraction. The molecule serves as the photocathode and the laser field as the accelerating field for a "self-diffraction" measurement. This new technology is now called laser-induced electron diffraction (LIED).
The recolliding electron can elastically (as discussed above) or inelastically scatter from its parent ion. Scattering is the realm of collision physics. Thus, collision physics and optical physics are united. Recollision brings something that is qualitatively new to each. Through recollision, optical methods hold the potential for structural determination. Through recollision, collision physics gains a systematic method for measuring collision dynamics and for controlling it (Niikura et al. 2002, 2003).

Before leaving the basic three-step recollision model, we return to the continuum wave packet. Approximately half of the wave packet that tunnels directly leaves the influence of the parent ion. These electrons provide access to pure tunneling information. As we know from other areas of science, tunneling can be a very important diagnostic, e.g., in microscopy (Bai 2000).

So far we have discussed how spectroscopy will be extended as a result of recollision physics. We have not discussed how the attosecond optical pulse itself can be used. Further, we have not discussed the extensions to spectroscopy that arise from the near-perfect synchronization between the attosecond pulse and the fundamental field, which is another unusual aspect of this new technology.

There are two ways by which high harmonics and attosecond pulses will impact spectroscopy. First, they are a laboratory source of broad bandwidth radiation extending over the region from $\sim 10$ to $300 \mathrm{eV}$ that is difficult to access by other means. The technology is well developed and it can now be used as an excitation source.

Compared with other sources, however, high-harmonic radiation does not naturally have narrow line width, but it is characterized by ultrashort pulse durations and a high degree of coherence. In order to produce high harmonics, population in both the bound state and the continuum is required. Since continuum population continually leaks away, it must be constantly repopulated. The only way by which this is possible is to use a relatively short fundamental pulse. In the future, high-harmonic frequency combs may allow the large bandwidth of short-pulse technology to be combined with narrow comb lines.

The most obvious impact of attosecond pulses is in measuring faster processes. Attoseconds are the natural timescale of electrons. Hence, attosecond technology gives access to measuring electronic dynamics involving valence electrons. Clearly, the long-term importance of developing methods to measure and control matter on this timescale is difficult to overestimate. At first glance this seems simple: just extend nonlinear spectroscopy from the optical range to the XUV. However, it is not nearly so simple. Boundcontinuum cross sections are small and attosecond sources are relatively weak. Therefore, conventional pump-probe experiments remain essentially impossible. The technology that has been developed for measuring the attosecond pulses 
themselves, or measuring the dynamics that they might excite, is closely related to the technology that is used to create the pulse in the first place, so we return to classical physics.

An electron that tunnels from a bound state to the continuum gains a phase-dependent momentum from the laser field that drove the tunneling. When we measure the electron, the process is often called above-threshold ionization. This term refers to the fact that the electron can gain much more than the minimum energy needed for ionization: a breakdown of the perturbation approximation.

Tunneling is only one method of releasing an electron into the continuum. Attosecond photoionization is another. In the case of photoionization, the phase-dependent electron momentum gained from the field measures the range of times over which the electrons were released. If a simple atom (such as hydrogen) is the ionized target, then we measure the duration of the attosecond pulse. This approach to measurement is called the attosecond streak camera. If a more complex system is the source of the photoelectrons, and the duration of the optical pulse is known, then we measure the dynamics of the medium.

In the following, we amplify some of the concepts and also discuss attosecond technology-how attosecond pulses are made and measured.

\section{STRONG FIELDS AND ATTOSECOND PHYSICS}

Attosecond physics emerged from the study of the interaction of intense laser pulses with matter. Focused femtosecond laser pulses at intensities of $\sim 10^{14} \mathrm{~W} \mathrm{~cm}^{-2}$ produce electric fields that are comparable to those experienced by valence electrons. In the presence of such fields, ionization produces a free electron that is subsequently accelerated by the oscillating laser field. Some of these electrons are driven back to the ionic core and can recollide with it. Recollision is accompanied by several possible phenomena that are illustrated in Figure 1. (i) The recolliding electron can scatter elastically off the core. These electrons can acquire a higher total kinetic energy than those that do not rescatter and are therefore easily identified experimentally. They carry diffraction information on the molecular structure as discussed later (Section 6.2). (ii) The recollision can also be inelastic. Inelastic scattering leads to excitation, fragmentation, or double ionization of the parent species (Section 5.2.1). (iii) Radiative recombination to the neutral species may occur, producing short-wavelength radiation (Section 4.1). In this process, the kinetic energy gained by the electron in the laser field is converted into light. All of the above phenomena are repeated every half-cycle of the

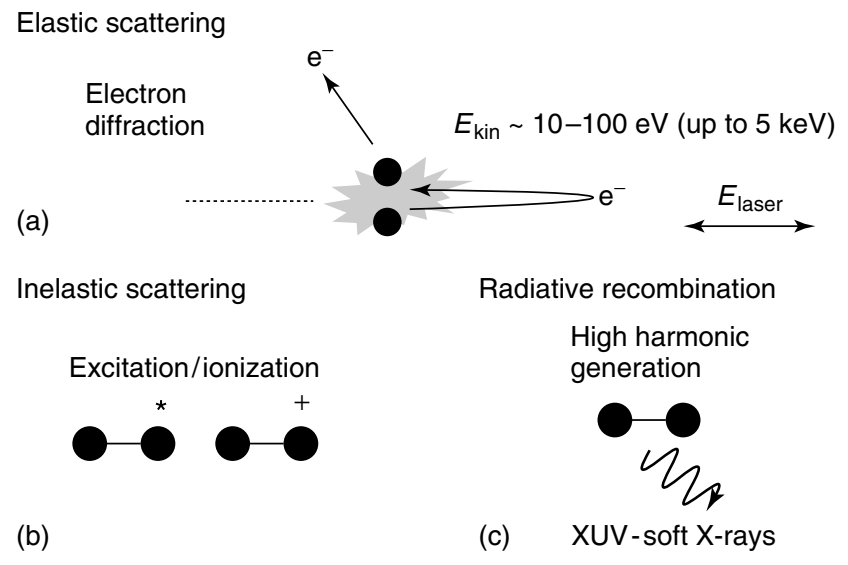

Figure 1 Schematic illustration of the processes induced by electron recollision in a molecule exposed to a strong laser field. The recolliding electron acquires a maximum kinetic energy of $3.17 \mathrm{U}_{\mathrm{p}}$ and can scatter (a) elastically or (b) inelastically off the ion core or (c) it can recombine under emission of electromagnetic radiation that extends into the $\mathrm{XUV} /$ soft-X-ray region.

driving laser field (i.e., every $1.33 \mathrm{fs}$ at $800 \mathrm{~nm}$ ) and occur within windows of a few hundred attoseconds.

Attosecond physics relies on the dynamics of electrons induced by a strong laser field. These dynamics are understood in terms of the three elementary steps: ionization, acceleration, and recombination, which are discussed later.

\subsection{Ionization in a Strong Laser Field}

All atomic or molecular ionization in an intense infrared (IR) pulse is multiphoton ionization. However, in common usage, ionization is usually classified into multiphoton or tunneling ionization, which are two limiting cases. Multiphoton ionization occurs preferentially in the limit of short wavelengths and low intensities, whereas tunneling ionization occurs in the limit of long wavelengths and high intensities. The former can be understood as the absorption of a finite number of photons, which results in ionization. In the absence of resonances, the ionization rate scales as

$$
r_{\text {multiphoton }} \propto I^{n}
$$

where $n$ is the number of photons and $I$ is the intensity of the laser field.

Tunnel ionization occurs if the laser field is strong enough to modify the coulomb potential sufficiently to allow the escape of an electron by tunneling and it varies slowly enough that the field can be approximated as a static field, time entering as a parameter. The coulomb potential of a hydrogenic atom and the combined coulomb and electrostatic potential of the laser field are represented 
in Figure 2. In the limit of tunnel ionization, the ionization rate scales as

$$
r_{\text {tunneling }} \propto \mathrm{e}^{-\frac{2}{3} \frac{I_{\mathrm{p}}^{3 / 2}}{E}}
$$

where $I_{\mathrm{p}}$ is the ionization potential and $E$ the field strength in atomic units.

The two processes are limiting cases and most experiments have characteristics of both. The transition between the two regimes is characterized by the Keldysh parameter:

$$
\gamma=\sqrt{\frac{I_{\mathrm{p}}}{2 U_{\mathrm{p}}}}
$$

where $U_{\mathrm{p}}$ is the ponderomotive potential of the laser field that is related to the electric field strength $E$ and the angular frequency $\omega$ of the latter as

$$
U_{\mathrm{p}}=\frac{E^{2}}{4 \omega^{2}}
$$

For $\gamma \gg 1$, multiphoton ionization is a more adequate description than tunnel ionization and for $\gamma \ll 1$ the opposite applies.

Most experiments using intense laser pulses with a wavelength of $800 \mathrm{~nm}$ or more take place at or near the tunneling limit $(\gamma \leq 1)$ and therefore tunneling captures the essential physical aspects in an intuitive manner. The phenomenon has been studied in detail theoretically by Perelemov et al. (1966) and subsequently by Ammosov et al. (1986) and Krainov (1997). The theory has been applied to describe the tunnel ionization of molecules (Tong, Zhao and Lin 2002). For this purpose, the orbital from which ionization occurs is expanded in a series of atomic orbitals localized at the center of charge of the molecule and their contributions are summed coherently.

After ionization, the electron emerges in the continuum, where it is subject to the strong laser field. To describe the evolution of the electron in the continuum, knowledge of its initial momentum distribution is needed. After the electron leaves the tunnel, the momentum distribution in the direction perpendicular to the polarization of the laser field is not affected by the field. The final momentum distribution is thus simply given by the initial momentum distribution (i.e., the orbital in momentum space) multiplied by a "tunneling filter" that weights the initial distribution with the ionization probability determined by

$$
\Psi_{c, \perp} \propto \Psi_{\mathrm{b}, \perp} \exp \left(-\frac{p_{\perp}^{2} \sqrt{2 I_{\mathrm{p}}}}{2 E}\right)
$$

where $\Psi_{\mathrm{b}, \perp}$ is the perpendicular component of the boundstate momentum orbital from which ionization takes place, $p_{\perp}$ is the perpendicular component of the electron momentum, and $E$ is the electric field strength. In the direction parallel to the laser polarization, the final momentum distribution is affected by the details of the laser field and follows a more complex expression (Krainov 1997).

\subsection{Classical Propagation and Recollision}

After ionization, the electron propagates in the laser field. The motion of the electron wave packet is well approximated by classical equations. For simplicity, the coulomb potential is neglected, which is a valid approximation for large recollision energies since the laser electric field dominates the motion over the major part of the electron trajectory. This is called the strong-field approximation (SFA). Moreover, the initial velocity of the electron is small and often neglected. For a linearly polarized laser pulse with the time-dependent electric field amplitude $E(t)=E_{0} \cos (\omega t)$, the position as a function of time is given by

$$
\begin{aligned}
x(t)= & \frac{E_{0}}{\omega^{2}}\left[\cos \left(\omega t_{0}\right)-\cos (\omega t)\right. \\
& \left.+\omega\left(t_{0}-t\right) \sin \left(\omega t_{0}\right)\right]+x\left(t_{0}\right)
\end{aligned}
$$

where $t_{0}$ is the time of ionization. The time of recollision $t_{r}$ is obtained by solving the equation $x\left(t_{r}\right)=0$. The electrons
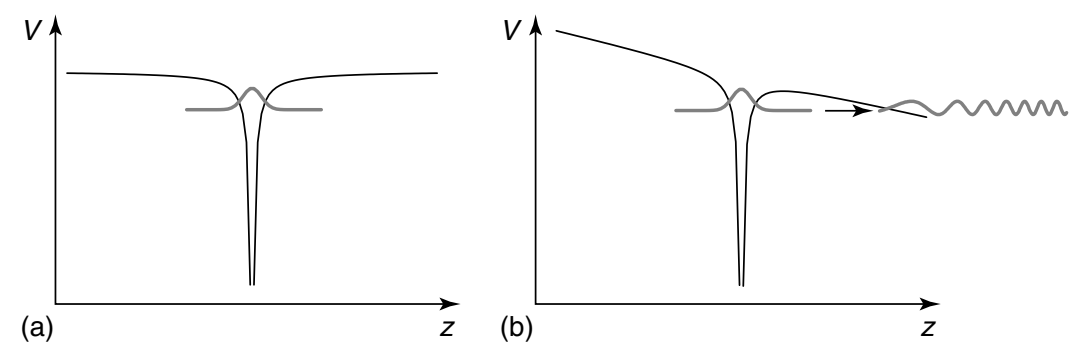

Figure 2 Illustration of a coulomb potential and the wave function of an electron in its lowest bound electronic state in (a) the absence of an external field and (b) a static electric field. If the external field is strong enough, the bound electron may escape by tunneling into the continuum as represented by the arrow in (b). 
that are ionized in the interval $-\pi / 2<\omega t_{0}<0$ or $\pi / 2<$ $\omega t_{0}<\pi$ (modulo $2 \pi$ ) are dragged away from the ion core by the oscillating laser field and never return. If tunnel ionization occurs at $-\pi<\omega t_{0}<-\pi / 2$ or $0<\omega t_{0}<\pi / 2$, the electron can return to the parent ion within the same laser cycle. In the case of a long laser pulse, similar ionization events occur at each field maximum and are thus repeated at half the laser period. The total kinetic energy acquired by the electron at the moment of recollision $E_{\text {kin }}=1 / 2 v^{2}$ is obtained from its final velocity:

$$
E_{\mathrm{kin}}=2\left(\sin \left(\omega t_{r}\right)-\sin \left(\omega t_{0}\right)\right)^{2} U_{\mathrm{p}}
$$

Figure 3 represents the laser electric field, the acquired kinetic energy as a function of the time of ionization and time of recollision, and the extension of the classical trajectory for a laser field with a wavelength of $800 \mathrm{~nm}$. The maximum total kinetic energy that a recolliding electron can acquire in the laser field is $3.17 U_{\mathrm{p}}$. For each total kinetic energy gained in the laser field, there are two possible trajectories, which are represented by arrows in Figure 3. One of the trajectories initiates in the interval $0<\omega t_{0}<17^{\circ}$ and recollides after $\omega t_{\mathrm{r}}>252^{\circ}$, whereas the other trajectory is born in the interval $18^{\circ}<\omega t_{0}<90^{\circ}$ and recollides at $\omega t_{\mathrm{r}}<252^{\circ}$. The two types of trajectories are called long and short according to the length of their transit time. Figure 3(c) represents the classical excursion amplitude as a function of the phase in the laser cycle. The electrons that are ionized while the electric field is increasing never return to the ion core and acquire a maximum kinetic energy of $2 U_{\mathrm{p}}$. Rescattered electrons can have an energy of up to $10 U_{\mathrm{p}}$ (Paulus et al. 1994).

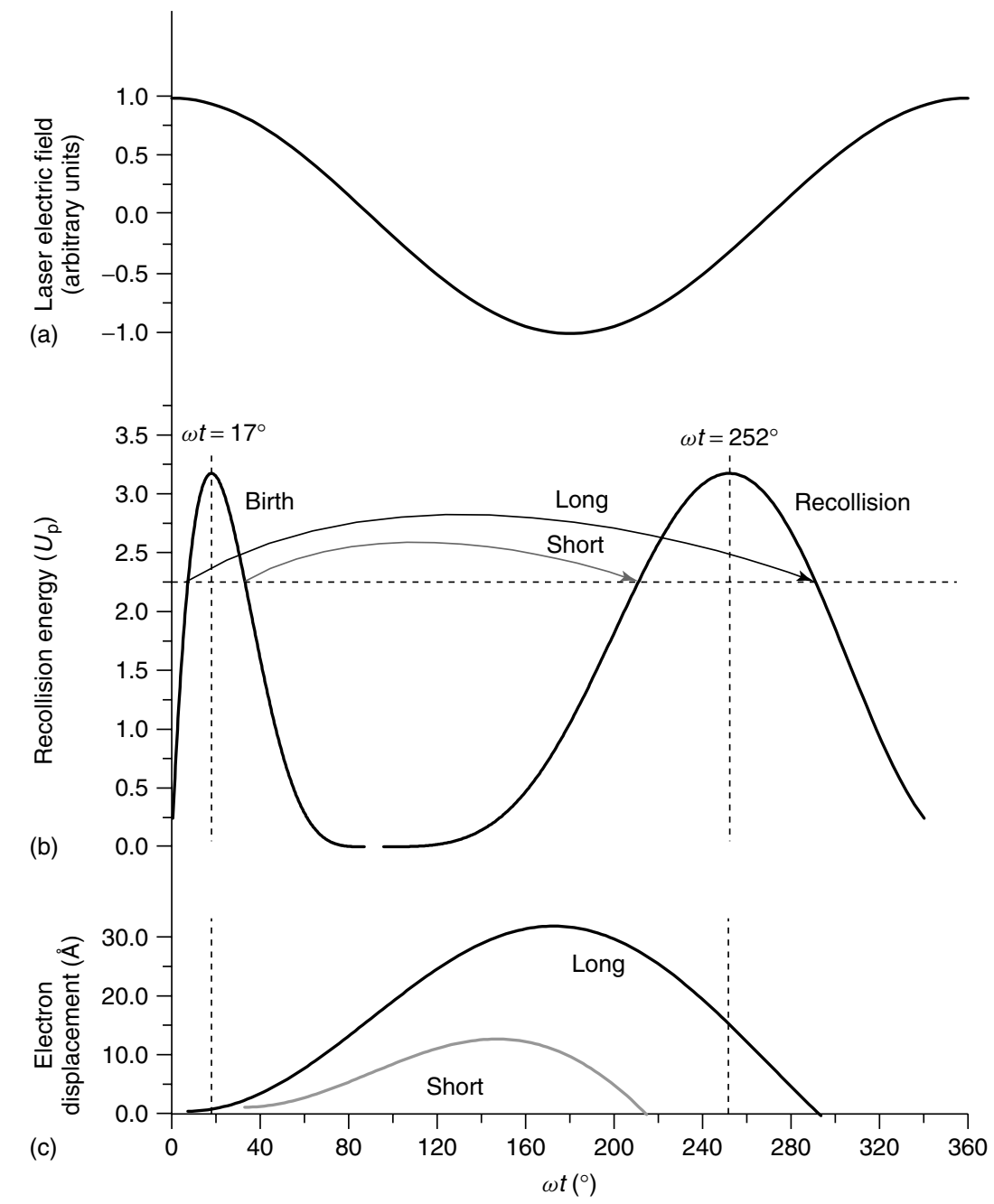

Figure 3 Results of classical calculations of electron recollision in a periodic laser field. (a) This represents the electric field $E(t)=\cos (\omega t)$ of the laser as a function of its phase $\omega t$. (b) This shows the kinetic energy of the electron at the moment of recollision as a function of its phase of birth (left-hand side curve) and its phase of recollision (right-hand side curve). The arrows highlight two possible trajectories "long" and "short" that correspond to the same recollision energy but differ in duration. (c) This represents their spatial excursion for a laser field of wavelength $\lambda=800 \mathrm{~nm}$ and intensity $I=3 \times 10^{14} \mathrm{~W} \mathrm{~cm}^{-2}$. 


\subsection{Quantum Mechanical Models}

The time-dependent Schrödinger equation (TDSE) provides the most accurate quantum mechanical description of the interaction between a strong laser field and a molecule. The results of simulations using the TDSE are often found to compare well with experimental data, but they provide little physical insight into the involved mechanisms. Another quantum mechanical description known as the Lewenstein model (Lewenstein et al. 1994) is very popular because it provides analytical solutions for the evolution of an atom in a strong laser field by neglecting the coulomb potential, i.e., using the so-called SFA.

The Lewenstein model concentrates on the XUV radiation that is emitted during the recollision. It shows how the quantum mechanical aspects modify the classical picture. The following approximations are made. (i) In the strong field, the excited bound states of the atom play no role and their population is neglected. (ii) The evolution of the continuum electron is not affected by the coulomb potential, which is therefore neglected. This leads to the following ansatz for the time-dependent wave function as a superposition of the ground-state wave function and a continuum wave packet:

$$
\begin{aligned}
\Psi & =\Psi_{\mathrm{b}}+\Psi_{\mathrm{c}} \\
& =a(t) \mathrm{e}^{\mathrm{i} I_{\mathrm{p}} t}\left|\Psi_{\mathrm{g}}\right\rangle+\int \mathrm{d}^{3} \vec{v} b(\vec{v}, t)|\vec{v}\rangle
\end{aligned}
$$

where $\left|\Psi_{\mathrm{g}}\right\rangle$ represents the ground-state wave function and $|\vec{v}\rangle$ represents the atomic state with one continuum electron of velocity $\vec{v}$. The time-varying dipole moment of the system is given by

$$
\begin{aligned}
\vec{d}(t)= & \left\langle\Psi_{\mathrm{b}}|\vec{r}| \Psi_{\mathrm{b}}\right\rangle+\left\langle\Psi_{\mathrm{c}}|\vec{r}| \Psi_{\mathrm{c}}\right\rangle+\left\langle\Psi_{\mathrm{b}}|\vec{r}| \Psi_{\mathrm{c}}\right\rangle \\
& +\left\langle\Psi_{\mathrm{c}}|\vec{r}| \Psi_{\mathrm{b}}\right\rangle
\end{aligned}
$$

where $\left|\Psi_{\mathrm{c}}\right\rangle$ represents the continuum part of the wave function of equation (8). The first term in equation (9) represents the ground-state dipole moment and vanishes in an atom. The second term represents the dipole generated by the acceleration of the continuum electron in the laser field. Its contribution is negligible compared to the last two terms representing the recombination of the continuum electron with the ion core to form the ground state. This term represents the process that leads to the emission of short-wavelength attosecond pulses. The spectrum of the emitted radiation is obtained from the Fourier transform of the dipole acceleration.

Neglecting the influence of the coulomb potential, the canonical momentum

$$
\vec{p}=\vec{v}+\vec{A}
$$

is a conserved quantity for the continuum electron, which leads to the following expression for the time-varying dipole moment:

$$
\begin{aligned}
d(t)= & i \int_{0}^{t} \mathrm{~d} t^{\prime} \int \mathrm{d}^{3} \vec{p} E_{0} \cos \left(t^{\prime}\right) d_{x}\left(\vec{p}-\overrightarrow{\left.A\left(t^{\prime}\right)\right)}\right. \\
& \times \exp \left[-i S\left(\vec{p}, t, t^{\prime}\right)\right] d_{x}^{*}(\vec{p}-\overrightarrow{A(t)})+c . c .
\end{aligned}
$$

where $d_{x}$ is the component of the dipole moment along the polarization direction of the laser field and

$$
S\left(\vec{p}, t, t^{\prime}\right)=\int_{t^{\prime}}^{t} \mathrm{~d} t^{\prime \prime}\left(\frac{\left[\vec{p}-\vec{A}\left(t^{\prime \prime}\right)\right]^{2}}{2}+I_{\mathrm{p}}\right)
$$

Equation (11) is an integral over probability amplitudes corresponding to the three steps that have been discussed above. Read from left to right, these terms stand for the transition at time $t^{\prime}$ from the ground state to the continuum state with canonical momentum $\vec{p}$. During the propagation in the continuum, the system acquires a phase factor $\exp \left[-\mathrm{i} S\left(\vec{p}, t, t^{\prime}\right)\right]$ depending on the classical action, the ionization potential, and the transit time $\Delta t=t-t^{\prime}$. In the last step, the electron recombines with the ground state at time $t$. Equation (11) is a high-dimensional integral but the dominant contributions come from regions of the integration space where the action $S$ is slowly varying

$$
\nabla_{\vec{p}} S\left(\vec{p}, t, t^{\prime}\right)=0
$$

which correspond to classical trajectories, thus establishing the link with the classical model.

\section{SYNTHESIS OF COLLISION AND OPTICAL PHYSICS}

As discussed in Section 2, light creates a coherent electron wave packet by tunnel ionization that recollides. During the recollision, the electron can elastically or inelastically scatter and create coherent light again (Figure 1). Thus, there is a unique interplay between optical and collision physics. Bringing both areas of science together leads to new methods for the investigation of ultrafast processes in atoms, molecules, and solids.

From a collision perspective, optics provides accurate timing of a collision because the laser field initiates the electron's initial release. This event has a highly nonlinear dependence on the instantaneous electric field and therefore it can be controlled on the subcycle timescale. Controlling the waveform of the optical field controls the creation of the free electron wave packet. 
From a collision perspective, optics also provides control. The continuum wave packet is controlled by the driving field on a subcycle timescale and the return also depends on the detailed structure of the driving field. The electron wave packet is manipulated by varying any of the usual laser parameters - amplitude, polarization, frequency, and bandwidth. Even greater control is possible by superimposing multiple frequencies.

Finally from a collision perspective, the recollision electron has unique properties. The recollision probability peaks very strongly about three-fourths of a period after ionization. From the ion's point of view, the collision probability is about the same as from an external beam with a current density of $10^{11} \mathrm{~A} \mathrm{~cm}^{-2}$ (Section 5.2.1). This is orders of magnitude higher than the current density used in typical collision experiments.

From an optics perspective, the de Broglie wavelength of the recolliding electron is in the range of $\lambda \sim 0.5-5 \AA$. This is ideal for imaging molecular dynamics. The short wavelength guarantees atomic scale resolution and the timing has attosecond accuracy. In the experiment, the scattered electron can be observed directly, as it can be converted to light where it can be read optically. During the conversion process, the recollision electron wave packet recombines with the ion core to produce the initial ground state. This phase matching can act as a filter, isolating this process against a background of incoherent processes. As we show in the following section, spatial information is preserved through this process - transferred from the coherent electron to a coherent photon.

The recollision process is followed by several possible mechanisms that have equivalents in other areas of molecular science. High harmonic emission is related to radiative recombination and can be considered to be a reverse photoelectron process. Recollision excitation, dissociation and ionization are equivalent to electron impact excitation, dissociation and ionization. In the case of the strong-field-driven process, the electron has a very broad spectrum and recollision occurs from a well-defined direction in space. This is fundamentally different from electron impact and scattering experiments where the electron kinetic energy distribution is kept narrow. This means that the collision experiment is performed over a very broad range of energies in every single laser shot. This advantage is exploited in high-harmonic generation (HHG) where the whole recombination spectrum is obtained at every recollision. Moreover, in the strong-field case, the recollision can be timed with attosecond precision (Section 5.2.1) and allows to study dynamical processes occurring on this timescale.

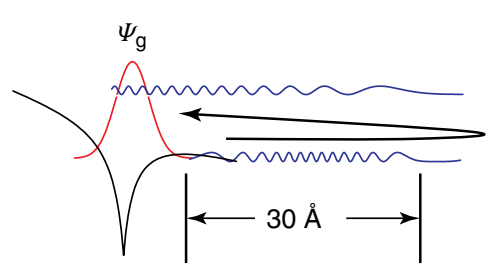

Molecular interferometer

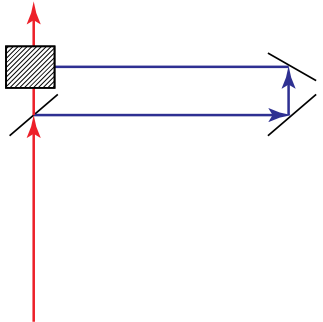

Optical interferometer
Figure 4 Illustration of the analogy between high harmonic generation and optical interferometry. Optical interferometry permits the characterization of two beams through their interference. In a similar manner, the properties of the high harmonics emitted at the moment of recollision can be used to characterize both the bound-state wave function and the continuum electron.

\subsection{Recollision and Electron Interferometry}

The process from tunnel ionization to recombination can be described in the language of interferometry. Optical interferometry is very powerful in its capabilities of completely characterizing the two interfering waves. We will show how the principles of interferometry can be applied to the complete characterization of the processes governing the birth of attosecond pulses. The strong field creates the superposition state of equation (8), which is a coherent superposition of the ground state and a continuum state. Tunnel ionization thus acts as a beam splitter that would split an optical beam in two. Figure 4 illustrates this analogy. The propagation of the electron in the laser field is analogous to the propagation of the secondary beam along one arm of the Michelson interferometer. Controlling the electron wave packet by shaping the external field is equivalent to modifying one arm of the interferometer. The final step is the recombination of the ionized system to form the neutral ground state under emission of a photon. This process is equivalent to the recombination of the two beams, which results in their interference. In the present context, the returning electron wave packet interferes with the remaining groundstate wave function and generates an oscillating dipole that emits the high harmonic radiation. The emitted light contains the complete information on the recolliding electron wave packet and the ground-state wave function and can be used to characterize either of them as we show below.

\subsection{Coherent Conversions between Photons and Electrons}

The process of recombination is described as a dipole transition between a continuum state and the ground state of a molecule. A useful qualitative picture of the recollision 
wave packet is provided by a superposition of plane waves:

$$
\left|\Psi_{\mathrm{c}}\right\rangle=\int \mathrm{d} k a(k) \mathrm{e}^{\mathrm{i} k x}
$$

where $a(k)$ is a complex form factor and $x$ is the direction of propagation of the wave packet. Using this approximation, the matrix element for the emission of radiation is

$$
\vec{d}_{\mathrm{r}} \propto \int \mathrm{d} k a(k)\left\langle\Psi_{\mathrm{g}}|\vec{r}| \mathrm{e}^{\mathrm{i} k x}\right\rangle
$$

The phase of the emitted radiation is determined by the phase of $\vec{d}_{\mathrm{r}}$ and the instant of emission. The amplitudes and phases taken under different recollision angles allow a complete characterization of $\left|\Psi_{\mathrm{g}}\right\rangle$ (Section 6.1).

This process can be compared to photoelectron emission as it is done in traditional photoelectron spectroscopy. In classical photoelectron spectroscopy, the molecule is exposed to a spectrally narrow source of XUV radiation, which leads to the emission of photoelectrons carrying a kinetic energy that corresponds to the energy difference between the XUV photon and an interval between eigenstates of the neutral molecule and the cation. This kinetic energy is measured and provides information on the level structure of the molecular cation or, equivalently, the molecular orbital structure of the neutral molecule (Kimura et al. 1981). The photoelectron angular distributions (PADs) provide additional information on the spatial structure of the molecular orbital from which ionization takes place. Molecular frame PAD (MFPAD) are highly sensitive to the structure of the orbital from which the electron was ionized, a property that has been successfully exploited in understanding photodissociation processes (Gessner et al. 2006).

The process of recombination following tunnel ionization and propagation in a strong laser field can be viewed as a process complementary to photoelectron emission, which is governed by the same matrix elements. The continuum state is, however, radically different since it is spectrally very broad and recollision takes place from a well-defined spatial direction. The similarity of the involved matrix elements implies that the information content is similar but the way of extracting it is very different. There are several key features of the strong-field process that make it attractive for the investigation of molecular structure and dynamics. In photoelectron emission, the ionized molecule emits a photoelectron over the full solid angle. Space charge effects severely restrict the maximal signal by degrading spectral resolution. In strong-field recombination, the kinetic energy of the recolliding electron is converted into photons that are emitted in a highly collimated beam copropagating with the fundamental laser beam. Since the electron is in the continuum for only a few femtoseconds, there is no problem with space charge. The recolliding electron comes in short bursts that are precisely timed and last only a few hundred attoseconds. This feature permits the application of the process to the study of ultrafast molecular processes as discussed later. The broad spectrum of the recolliding electron means that information is available for a very large range of energies in a single experiment. Since molecules can be aligned in space (Itatani et al. 2005) and recollision takes place from the direction imposed by the laser polarization, the recolliding electron can probe the molecular structure from different angles relative to the molecular axis. Combined with the short de Broglie wavelength of the electron $(\sim 1 \AA)$, it implies that molecular imaging on an attosecond timescale is possible. The main disadvantage of high-harmonic generation compared to photoelectron detection is that the process to be observed takes place in a strong laser field. The latter may modify the electronic structure by polarization or even induce complex nonadiabatic dynamics, especially in larger molecules (Lezius et al. 2001, 2002, Smits, de Lange, Stolow and Rayner 2004).

\section{ATTOSECOND PULSES, MEASUREMENT, AND CONTROL}

\subsection{High-harmonic Generation and Attosecond Pulse Trains}

A typical high-intensity femtosecond laser pulse $(800 \mathrm{~nm}$, $30 \mathrm{fs}$ ) consists of many optical cycles. Consequently, the processes of tunnel ionization, acceleration, and recombination are repeated twice per period of the oscillating field and therefore the XUV radiation is emitted in short bursts spaced by half the laser period, i.e., $1.33 \mathrm{fs}$ at $800 \mathrm{~nm}$. The spectrum of such a train of attosecond pulses is periodic with twice the photon energy. Since the dipole operator that governs recombination is odd under inversion, the emitted frequencies are odd integer multiples of the frequency of the driving field. The first observation of attosecond pulse trains was achieved in the frequency domain by dispersing the light produced by focusing a femtosecond laser pulse into a rare gas target (McPherson et al. 1987, Ferray et al. 1988). The nonperturbative nature of the process was immediately recognized because the efficiency of harmonic generation remains nearly constant over a broad range of photon energies (the so-called "plateau") before falling off abruptly in the "cut-off" region. This phenomenon is well understood in terms of the three-step model discussed in Section 2.2.

The lower limit of the energy bandwidth of individual harmonics is determined by the length of the driving pulse. In the limit of a very short driving pulse, there is only a single energetic recollision and the high-energy emission has a continuous spectrum. In the opposite limit, 
a comb of narrow odd harmonics is obtained. However, the driving laser pulse cannot be made arbitrarily long because the medium requires ground-state molecules and simultaneously a continuous supply of continuum electrons. Harmonic emission must therefore end. Frequency combs offer a means to combine short-pulse requirements with the narrow linewidth needed for precision spectroscopy (Section 4.4).

The basic idea behind almost all attosecond pulse measurements is to use the pulse to produce an electron replica and measure the replica instead. Photoelectrons produced by ionizing a simple atom can serve this purpose. Their spectrum, amplitude, and phase are determined by the attosecond pulse and the known transition moment.

We have described above how high harmonic generation, when viewed in time, is a train of attosecond pulses spaced by half the laser period. The first experimental characterization of an attosecond pulse train has been realized by Paul et al., who implemented the so-called RABBITT (Paul et al. 2001) technique (reconstruction of attosecond beating by interference of two-photon transitions). It consists in reconstructing the electric field $E(t)$ of the pulse train by measuring the spectral amplitude $E(\omega)$ and the relative phase $\phi(\omega)$ of the harmonics. The attosecond pulse train is overlapped with the residual IR laser pulse $\left(10^{11}-10^{12} \mathrm{~W} \mathrm{~cm}^{-2}\right)$ that has been used to generate the XUV radiation in a gas jet. The atoms are used to produce a photoelectron replica of the attosecond pulse train, which is modified by the IR field. Since the latter is rather weak, the interaction is best described in terms of absorption of photons. The observed photoelectron spectrum is dominated by peaks corresponding to ionization by the odd harmonic components that are spaced by twice the fundamental photon energy. The IR field leads to the appearance of sidebands corresponding to absorption or stimulated emission of one IR photon. Each sideband possesses two interfering contributions from the two adjacent harmonics. Observation of the intensity of the sidebands and their angular distribution permits the extraction of the relative phase of the two harmonics. The procedure thus provides the relative phase of all harmonics, which allows the reconstruction of the electric field $E(t)$ of the pulse train. The application to pulse trains generated from argon, krypton, or xenon (Aseyev et al. 2003) has shown that the timing of the harmonic emission depends on the atom that is used to generate the harmonics. While in argon the emission takes place in the vicinity of the zero-crossing of the IR electric field as expected from the three-step model, it occurs significantly earlier in xenon. Mairesse et al. have used the RABBITT technique to measure the chirp of an attosecond pulse train over a broad spectral range and have generated 150-as pulses from neon by optimizing the spectral bandwidth (Mairesse et al. 2003).

\subsection{Single Attosecond Pulses and Measurement}

The most obvious way of performing attosecond measurements is to use a pump-probe scheme in which the first pulse initiates the dynamics one wishes to observe and the second pulse acts on the system to produce an observable signal that carries information on the state of the system. Such measurements rely on the capability of producing single pulses of attosecond duration. There are two main ways to achieve this goal. The first exploits the high sensitivity of the recollision probability to the polarization state of the driving laser field. The recollision probability is maximal for linear polarization of the driving field but it quickly decreases with increasing ellipticity because the electron is driven away from the ion core, thus preventing efficient recombination. A single attosecond pulse can be generated by quickly changing the polarization state during the pulse. The pulse starts in circular polarization and quickly sweeps through linear polarization back to circular polarization (Corkum 1994). Ideally, the linear polarization state only lasts for one laser cycle, permitting a single efficient recollision. This scheme has been realized experimentally (Kovacev et al. 2003) and has been used to produce some of the shortest attosecond pulses to date, lasting only 130 as (Sansone et al. 2006).

The second way of generating single attosecond pulses is to reduce the duration of the driving pulse so far that it only induces a single energetic recollision. This principle is illustrated in Figure 5. For both cases, the phase of the electric field oscillation with respect to the envelope (the so-called carrier envelope phase (CEP)) plays an important role. The electric field is given by

$$
E(t)=E_{e}(t) \cos (\omega t+\phi)
$$

where $E_{e}(t)$ is the pulse envelope function and $\phi$ is the CEP. If the CEP is close to zero, the driving field has a single pronounced maximum, which induces a single recollision with high kinetic energy. If the CEP adopts a value close to $\pi$, two identical recollisions can be achieved, thus producing a pair of attosecond pulses (Baltuska et al. 2003). The isolation of these attosecond pulses is realized through filtering of the short-wavelength radiation. In typical experiments employing neon as a generation medium, the selected photon energy is centered around $90 \mathrm{eV}$ (Drescher et al. 2002, Goulielmakis et al. 2008).

The very broad spectrum of a single attosecond pulse excludes the observation of photoelectron sidebands that is used in the RABBITT technique (Section 4.1). Its measurement, however, relies on a closely related method, the attosecond streak camera (Itatani et al. 2002, Kienberger et al. 2003). Again, the attosecond pulse generates a photoelectron replica in an atomic medium. As in the 


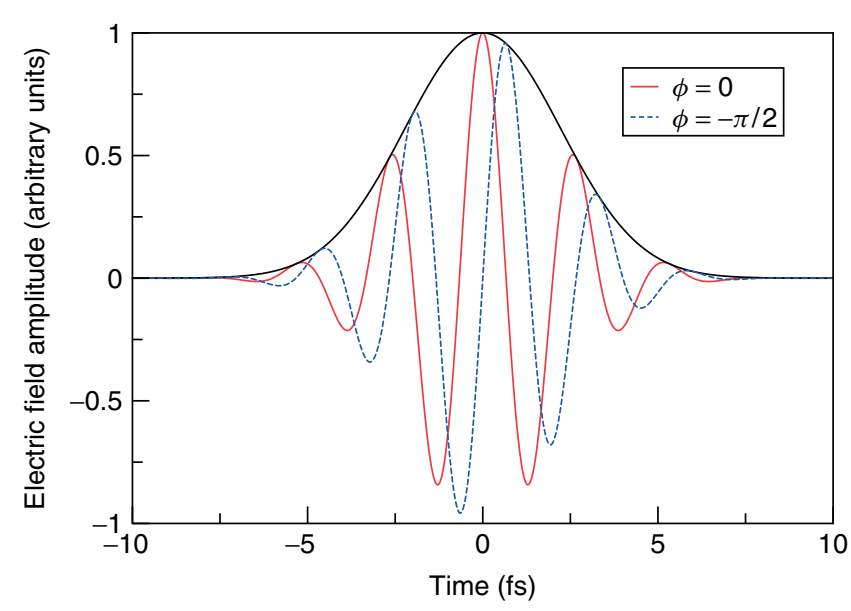

Figure 5 Envelope function and electric fields for two few-cycle pulses with a different value of the carrier envelope phase $\phi$.

RABBITT method, the residual IR field is superimposed with the XUV field but the IR intensity is high $\left(10^{13}-\right.$ $10^{14} \mathrm{~W} \mathrm{~cm}^{-2}$ ). In this case, the action of the IR field on the photoelectrons is better understood in terms of an acceleration. The velocity of the electrons is given by

$$
v(t)=-A_{L}(t)+\left[v_{0}+A_{L}\left(t_{i}\right)\right]
$$

where $v_{0}$ and $A_{L}\left(t_{i}\right)$ are the electron velocity and laser vector potential at the instant of ionization, respectively, and $v(t)$ and $A_{L}(t)$ are the corresponding quantities at a later time. As the electron is born in the continuum, it experiences an acceleration in the IR laser field, which modifies its velocity. The amount of this shift depends on the time when the electron is released. The time of birth of the electron in the continuum is mapped onto a final velocity that depends on the vector potential of the laser. The attosecond streak camera has been applied to the first characterization of an isolated 650-as pulse (Kienberger et al. 2003) and recently to the observation of the shortest attosecond pulse of 80-as duration (Goulielmakis et al. 2008).

\subsection{Controlling Attosecond Pulse Generation}

The attosecond streak camera and RABBITT are now the conventional methods of measuring attosecond pulses but the reader may recall that it is possible to think of attosecond pulse generation as a form of electron interferometry - a sheared interferometer. This opens a new opportunity for attosecond measurements-measure the recollision electron directly in the medium in which it was produced.

As we have seen, the recollision electron is a preexisting photoelectron replica translated into the attosecond pulse through the transition moment. Measurement is achieved by manipulating the arms of the interferometer. One method to do this is to add a weak second harmonic beam to the fundamental beam that produces the attosecond pulse. Modifying its phase relative to the fundamental allows us to scan the arms of the interferometer. All information needed to fully characterize the attosecond pulse is imposed on the spectrum of the attosecond pulse or pulse train (Dudovich et al. 2006).

It is interesting to consider how different this is from a conventional measurement. A high-order nonlinear process is hardly perturbed by adding another photon. Yet that added photon can impose an indelible imprint on the process (pulse), sufficient for measurement or even for control. This is likely to be a general characteristic of highorder nonlinear processes.

\subsection{Precision Measurements Using High Harmonic Radiation}

Frequency combs are well-established instruments in precision spectroscopy. They have recently become broadly available in the optical regime and open new perspectives for precision measurements of fundamental constants (Cundiff et al. 2001). The technology has been extended recently by the groups of Hänsch and Ye (Gohle et al. 2002, Jones et al. 2005). A buildup cavity enhances the output of a mode-locked femtosecond oscillator. The intensities that are reached at an intracavity focus are sufficient to generate harmonics in xenon or krypton atoms and the resulting XUV radiation is extracted using a broad-band output coupler. The coherence of the femtosecond pulse train traveling in the mode-locked oscillator is transferred into the XUV domain that offers attractive prospects for precision measurements at XUV frequencies and the investigation of coherence properties of high harmonic generation. Precision measurements at vacuum-ultraviolet and shorter wavelengths have become accessible only recently (Witte et al. 2005) and are highly relevant for testing the predictions of quantum electrodynamics or determining more accurate values of the proton-to-electron mass ratio. The coherence properties of HHG could be studied in much greater detail than before, allowing in particular for an accurate determination of the phase of emission of long and short trajectories.

\section{OBSERVATION OF ATTOSECOND DYNAMICS}

\subsection{Pump-probe Experiments}

The ultimate goal of attosecond spectroscopy is the imaging of electronic dynamics in real time. The most direct 
approach is to perform a pump-probe experiment as in traditional ultrafast spectroscopy (Zewail 1994). However, the intensity of the attosecond XUV pulses generated to date is not sufficient to perform these kinds of experiments with a reasonable signal level. In the future, such experiments may become feasible if the existing proposals on producing attosecond XUV pulses from free electron lasers can be put into practice (Zholents and Fawley 2004). So far, only theoretical results exist on XUV pump-XUV probe measurements, but the prospects are very promising $(\mathrm{Hu}$ and Collins 2006, Morishita et al. 2007, Schweigert and Mukamel 2007).

However, there is an alternative to XUV pump-XUV probe schemes, which consists in using the perfectly phased IR field as a pump or probe pulse. The most popular methods combine an isolated XUV pulse with the residual of the few-cycle IR pulse that was used for its generation. Two main methods are used in the characterization of ultrafast electronic processes: streaking and tunneling. An example of application of each technique is given below. Figure 6 illustrates the attosecond probing of electron dynamics. The high energy of the XUV pulses generated in neon (about $90 \mathrm{eV}$ ) has permitted the investigation of atomic dynamics initiated by inner-shell ionization (Kienberger et al. 2003, Uiberacker et al. 2007) but the availability of lower energy pulses (around $30 \mathrm{eV}$, (Sansone et al. 2006)) will also allow the study of molecular valence shell ionization dynamics.

\subsubsection{Attosecond Streaking}

The first application of attosecond pulses to the observation of electronic dynamics in real time was reported in 2002 (Drescher et al. 2002). This work showed that the emission time of Auger electrons produced following the inner-shell 3d ionization of krypton atoms can be measured with attosecond resolution using a time-to-energy mapping induced by a strong IR field. Krypton atoms were photoionized with a 900 -as pulse centered at $97 \mathrm{eV}$. The ionization of $3 \mathrm{~d}, 4 \mathrm{~s}$, and $4 \mathrm{p}$ electrons (processes a and $\mathrm{b}$ in Figure 6) is nearly instantaneous on the timescale of the XUV pulse and produces electrons that are identified by photoelectron spectroscopy. If ionization produces a $3 \mathrm{~d}$ hole, an electron from the $4 \mathrm{~s}$ shell can fill the vacancy, thereby ejecting an

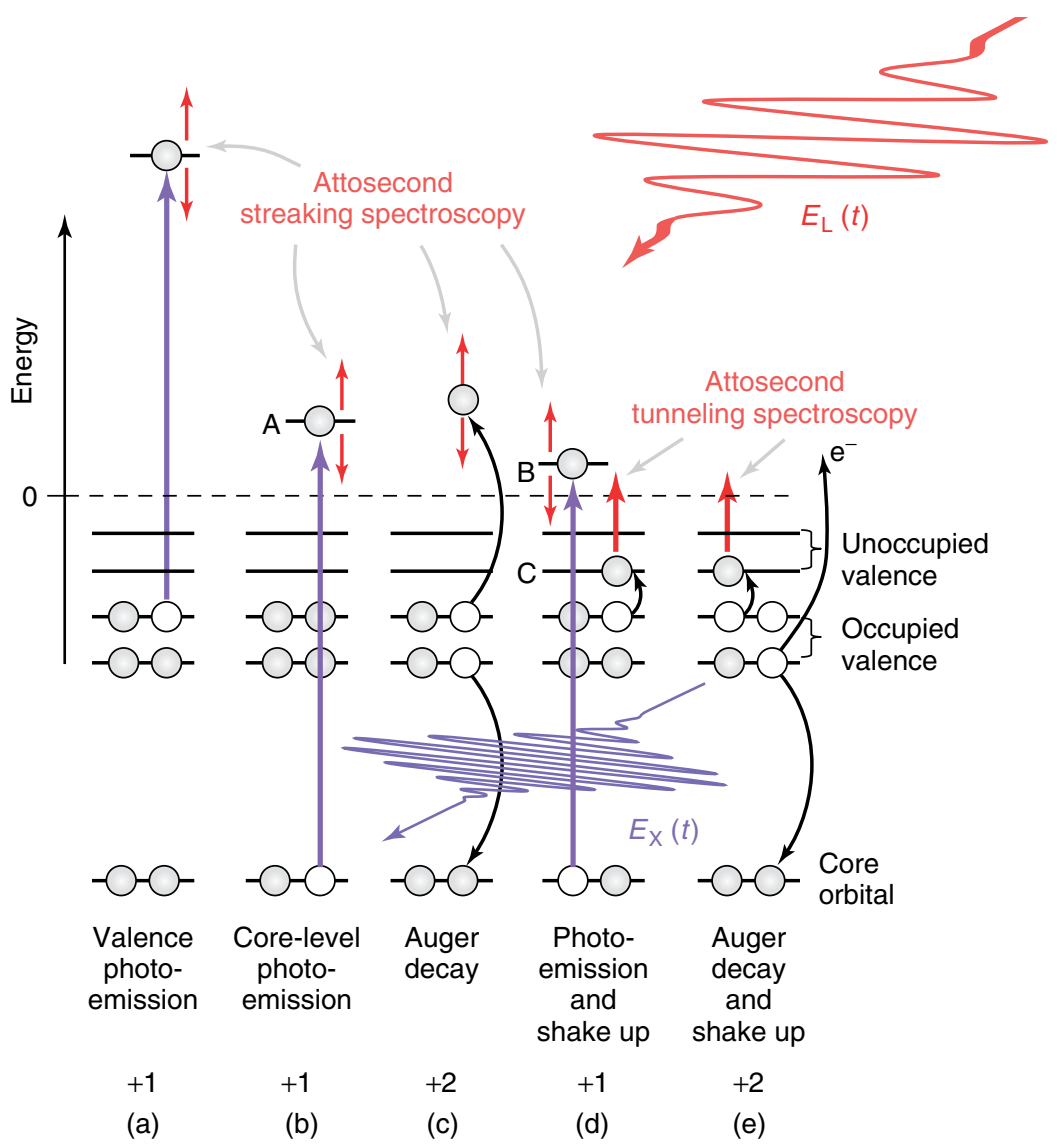

Figure 6 Electronic processes induced by an XUV pulse $\left(E_{X}(t)\right)$ in atoms and molecules and possible ways of tracing the induced electronic dynamics in real time using a few-cycle infrared pulse $\left(E_{\mathrm{L}}(t)\right)$. The labels +1 and +2 designate the final charge state of the ion. [Reproduced from Corkum and Krausz (2007) with permission.] 
electron from the $4 \mathrm{p}$ shell in a process known as the Auger decay. The latter process is slower than the duration of the XUV pulse and was observed in real time by Drescher et al. (2002).

The photoionization is performed in the presence of a strong IR field that imparts a momentum shift on the emitted electrons. The magnitude and sign of the momentum shift depend on the moment of ionization with respect to the phase of the IR field in the same way as described in Section 4.2. Assuming a linearly polarized light field $E(t)=E_{e}(t) \cos (\omega t+\phi)$, the final momentum of an electron released at time $t_{r}$ is changed by $\Delta p\left(t_{r}\right)=$ $\left[e E_{e}\left(t_{r}\right) / \omega\right] \sin \left(\omega t_{r}+\phi\right)$ along the direction of the electric field. Thus, if ionization occurs on a timescale that is short compared to the period of the IR field and the CEP is stable over the duration of the measurement, the observed electron spectrum will be shifted in energy by an amount that is periodic in the delay time between the XUV and IR pulse with the periodicity of the fundamental field. If, on the other hand, the emission is slow compared to the IR period, the electron spectrum will be characterized by sidebands appearing during the emission time. This is the case for the Auger decay of krypton. Drescher et al. (2002) measured the Auger electron spectrum for different delay times between the XUV and IR pulses and analyzed the area of the sidebands produced by the action of the IR field. The decay of the sideband area with delay time provides the lifetime of the $\mathrm{M}\left(d_{5 / 2}\right)$ vacancy of $7.9_{-0.9}^{+1.0} \mathrm{fs}$, which is in good agreement with frequency-domain measurements.

\subsubsection{Attosecond Tunneling}

Two other essential aspects of real-time electronic dynamics have recently become accessible with the introduction of the attosecond tunneling technique: light-induced tunnel ionization and the transient population of excited states as a result of electron correlation (Uiberacker et al. 2007). The attosecond tunneling method also relies on the combination of an attosecond XUV pulse and a strong few-cycle IR pulse but it addresses weakly bound atomic states that are transiently populated following XUV photoionization. In processes (d), (e), and (f) illustrated in Figure 6, one electron is ionized while another one is excited (the socalled shake-up), transiently populating an excited state that is subsequently field ionized under the action of the IR field. The method allows the tunneling of electrons from excited states to be observed in real time and cascaded Auger decay processes to be followed.

The experiments reported in Uiberacker et al. (2007) concentrated on the observation of tunnel ionization from shake-up states produced by $2 \mathrm{p}^{-1}$ ionization of $\mathrm{Ne}$ by a 250-as XUV pulse centered at $91 \mathrm{eV}$. The ionization is accompanied by the excitation of a second electron from the $2 \mathrm{p}$ shell into a higher lying orbital $n \ell$ (where $n=3,4$, $\ell=\mathrm{s}, \mathrm{p}, \mathrm{d})$. The experiment consists in measuring the yield of $\mathrm{Ne}^{2+}$ ions as a function of the delay between the XUV and the IR pulse. Varying the delay from negative to positive values shows a stepwise increase in the $\mathrm{Ne}^{2+}$ ion count as a result of the IR field reaching the threshold for tunnel ionization of a shake-up state. The signal increase is not smooth with delay time but exhibits sharp steps covering a range of 400 as around each field maximum of the IR pulse. The measurement is in good agreement with nonadiabatic theories of tunnel ionization (Yudin and Ivanov 2001) and constitutes the first experimental observation of the subcycle variation of the ionization rate.

The application of the method to xenon has led to the time-domain observation of cascaded Auger decay processes. Photoionization by the attosecond pulse centered at $91 \mathrm{eV}$ mainly ionizes the $4 \mathrm{~d}$ shell and also produces shake-up satellites of $4 \mathrm{~d}^{-1} 5 \mathrm{p}^{-1} n \ell$ configuration. All of these intermediate states of $\mathrm{Xe}^{+}$are transient and undergo a first Auger decay to several configurations of $\mathrm{Xe}^{2+}$ with a time constant A1, which in turn decay to the ground state of $\mathrm{Xe}^{3+}$ with a time constant A2. Varying the delay between XUV and IR field shows that the $\mathrm{Xe}^{3+}$ signal increases from zero at negative delay to a constant count rate at positive delays with a rise time of $6.0 \pm 0.7 \mathrm{fs}$, which is in good agreement with frequency-domain measurements. This laser-induced increase in the $\mathrm{Xe}^{3+}$ signal is due to IR tunnel ionization of the weakly bound states of $\mathrm{Xe}^{2+}$ and, therefore, measures the time constant A1. The count rate of $\mathrm{Xe}^{4+}$ ions increases with the same time constant as the $\mathrm{Xe}^{3+}$ signal but it exponentially decays at larger positive delays. $\mathrm{Xe}^{4+}$ ions are formed by IR-field double ionization of the high lying $\mathrm{Xe}^{2+}$ states of configuration $5 \mathrm{p}^{-4} n \ell n^{\prime} \ell^{\prime}$ and the decay therefore measures the Auger decay of these states to form $\mathrm{Xe}^{3+}$ in the ground state. The measured time constant amounts to $30.8 \pm 1.4 \mathrm{fs}$.

The attosecond tunneling method is therefore a useful tool to verify tunnel ionization theories and to observe a variety of multielectron dynamics directly in the time domain.

\subsection{Sublasercycle Dynamics}

\subsubsection{Nuclear Dynamics}

A complementary approach to observe processes on the attosecond timescale exploits the accurate timing of recollision with respect to ionization that is fully controlled by the strong driving field. In this case, the medium subjected to the strong field is the object under study. Measurements can reach attosecond precision in the time domain and ångström resolution in the spatial domain. The method 


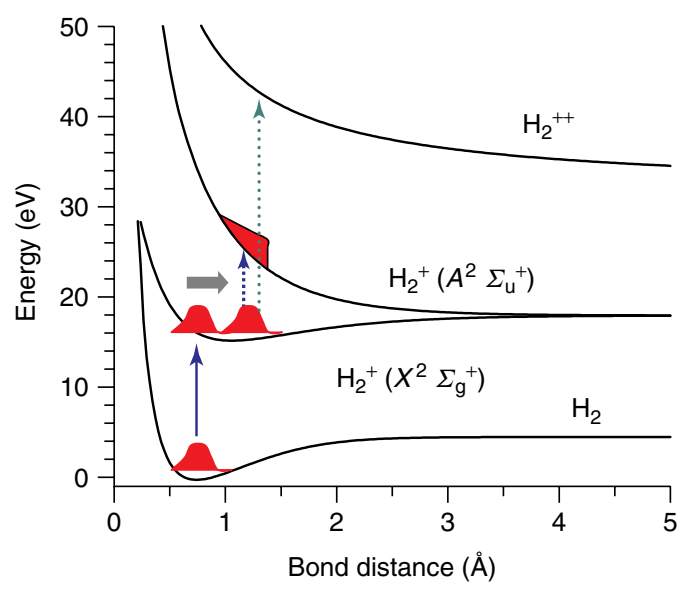

Figure 7 Relevant potential energy curves for $\mathrm{H}_{2}$ and its ions. Ionizing $\mathrm{H}_{2}$ forms two wave packets. One is a vibrational wave packet on the ground $\left({ }^{2} \Sigma_{\mathrm{g}}^{+}\right)$state of $\mathrm{H}_{2}^{+}$(shown). The other is an electron wave packet that moves in the laser field. The vibrational wave packet evolves until $\mathrm{H}_{2}^{+}$is excited by inelastic scattering caused by the returning electron wave packet. [Reproduced from Niikura et al. (2002) with permission.]

exploits the correlation between the ionized electron wave packet and the wave function of the ionic core to reach this unique accuracy. Instead of using attosecond photons that are generated in an external process, the attosecond electron pulses generated by tunnel ionization and accelerated by the laser field are used as a probe. Tunnel ionization triggers the dynamics by creating a nonstationary ion state whose subsequent evolution is investigated. Tunnel ionization (confined to $\sim 1 / 30$ of a period) is much faster than all vibrational motion in molecules and some electronic motions. The nuclei have no time to rearrange from the neutral equilibrium geometry to the ionic one. Consequently, tunnel ionization launches a vibrational wave packet that evolves until the time of recollision when it is probed by the returning electron. The observation of either fragmentation (possibly preceded by excitation) (Niikura et al. 2002, 2003) or XUV emission (Lein 2005, Baker et al. 2006) permits the characterization of the evolved system.

The first experiments exploiting correlation (Niikura et al. 2002, 2003) have shown that the technique accurately characterizes both the continuum electron wave packet and the vibrational wave packet of the molecular ion produced by strong-field ionization. In Niikura et al. (2002), hydrogen molecules were tunnel ionized by a 50 -fs IR laser pulse at an intensity of $1.5 \times 10^{14} \mathrm{~W} \mathrm{~cm}^{-2}$. Ionization, represented by the vertical arrow in Figure 7, simultaneously forms two wave packets: a vibrational wave packet moving on the field dressed $\mathrm{H}_{2}^{+}\left(\mathrm{X}^{2} \Sigma_{\mathrm{g}}^{+}\right)$surface and an electron wave packet moving under the effect of the laser field. As the electron wave packet returns to the parent ion, its inelastic scattering may cause excitation of the molecule or ionize it further, both processes leading to the dissociation of the molecule. The kinetic energy of the protons can be used to extract the internuclear separation at the moment of recollision and therefore allows a precise timing of the dissociation. The vibrational motion of $\mathrm{H}_{2}^{+}$is the clock that is started by tunnel ionization and is read out by fragmentation. The effect of the electric field on the potential energy surfaces is minimized by selecting molecules perpendicular to the electric field axis of the laser pulse in the detection step.

The experiment accurately characterizes the properties of the recolliding electron wave packet. The channels corresponding to recollision-induced processes are identified by the high kinetic energy of the proton fragments and their sensitivity to ellipticity of the laser pulses (see also Dietrich et al. 1994). With increasing laser ellipticity, the electron wave packet acquires increasing lateral momentum during the acceleration, which decreases its probability of recollision. The lateral displacement amounts to $7.7 \AA ̊$ under the conditions of the described experiment. This allows the determination of the velocity spread at the time of ionization to $\approx 4.2 \AA \mathrm{fs}^{-1}$. The time structure of the returning electron current density experienced by the ion is shown in Figure 8 . The current density reaches $8 \times 10^{10} \mathrm{~A} \mathrm{~cm}^{-2}$ in the second half-period after ionization, the most significant part being concentrated within a 1 -fs window. The time structure has been converted to a kinetic energy spectrum of the proton fragments and compared to the experimental results. The agreement confirms the calculated structure and shows that the first and third electron bunches dominate the observed kinetic energy spectrum.

These results reveal the unique properties of attosecond electron pulses to probe molecular dynamics. Exploiting correlation of wave packets, a 50-fs laser pulse is used to create an electron microbunch that has a duration of $1 \mathrm{fs}$ from the perspective of the correlated partner and reaches current densities that are not available otherwise. The actual observation of molecular dynamics using the same concepts followed in Niikura et al. (2003). The motion of a $\mathrm{D}_{2}^{+}$vibrational wave packet formed during tunnel ionization of $\mathrm{D}_{2}$ could be characterized over a span of several femtoseconds with a precision of 200 as and $0.05 \AA$. Since the tunnel ionized electron wave packet follows the oscillations of the driving laser field, the recollision time can be precisely controlled by varying the wavelength of the laser. The recolliding electron induces an excitation of $\mathrm{D}_{2}^{+}$from its $\mathrm{X}^{2} \Sigma_{\mathrm{g}}^{+}$ground state to the $\mathrm{A}^{2} \Sigma_{\mathrm{u}}^{+}$excited state that dissociates directly. The kinetic energy of the deuterons can be directly related to the internuclear separation of the molecular ion at the instant of recollision. Measuring the kinetic energy distribution of the fragments therefore provides a measure 


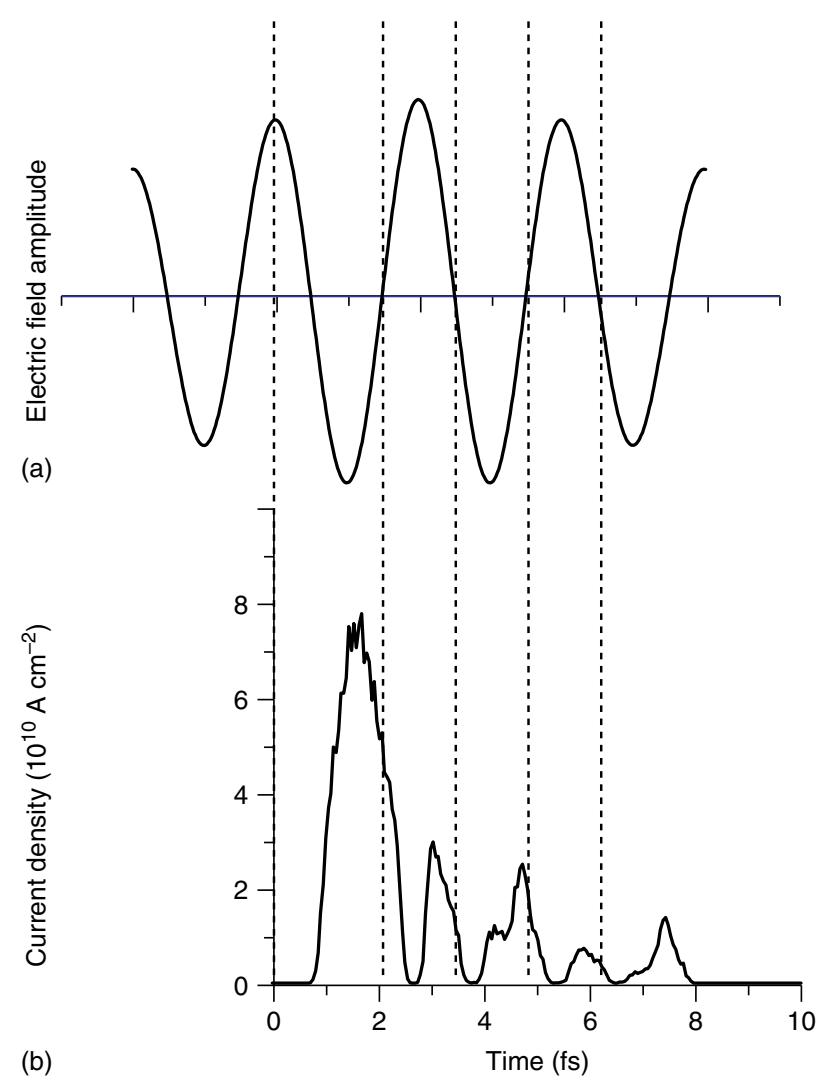

Figure 8 (a) Amplitude of the electric field of the laser pulse as a function of time. (b) Calculated electron current density experienced by the ion as a function of time after ionization. Only electrons with sufficient energy to induce $\mathrm{X}\left({ }^{2} \Sigma_{\mathrm{g}}^{+}\right) \rightarrow \mathrm{A}\left({ }^{2} \Sigma_{\mathrm{u}}^{+}\right)$ excitation by inelastic scattering are included.

of the internuclear separation as a function of recollision time. In the experiment, the influence of the laser field on the dynamics was minimized by selectively detecting fragments from molecules aligned perpendicular to the laser electric field axis. The experiment was repeated at four different wavelengths $800,1200,1530$, and $1850 \mathrm{~nm}$ that correspond to recollision times centered at 1.7, 2.7, 3.4, and $4.2 \mathrm{fs}$, respectively. For each wavelength, the most probable kinetic energy is converted into an internuclear separation using the field-free potential energy curves of $\mathrm{D}_{2}^{+}$, which amounts to effectively measuring the bond length of the cation at the recollision time. The results agree well with theoretical results on the motion of the vibrational wave packet and show that molecular dynamics can be monitored with attosecond resolution.

\subsubsection{Attosecond Strobing of Electronic Dynamics}

The observation of electronic dynamics in real time is one of the major goals of attosecond science. Turning from nuclear to electronic dynamics, tunnel ionization creates an ion in a nonstationary electronic state, which will therefore be subjected to electronic wave packet motion that can also be probed by the returning electron. The electronic motion triggered by an instantaneous ionization has been studied theoretically in a number of articles (see, e.g., Remacle and Levine 2006, Kuleff et al. 2005) but has not been observed experimentally. Nonstationary electronic states play an essential role in electron transport and charge transfer and their characterization in real time would hold significant promise in understanding these fundamental processes.

A recent experiment has shown that electronic dynamics in a simple two-level system can be probed using strong laser fields (Staudte et al. 2007). The upper state population could be mapped spatially within a time window of a few hundred attoseconds via coulomb explosion imaging. The experiment used cold target recoil ion momentum spectroscopy (Ullrich et al. 2003) to probe dynamics in $\mathrm{H}_{2}$ and $\mathrm{D}_{2}$ molecules. During the laser pulse with a duration of 40-140 fs, the neutral molecule is first tunnel ionized, which produces a vibrational wave packet in the $\mathrm{X}^{2} \Sigma_{\mathrm{g}}^{+}$ground state of $\mathrm{H}_{2}^{+}\left(\mathrm{D}_{2}^{+}\right)$. The wave packet then evolves toward the outer turning point of the vibrational motion where it moves into resonance with the threephoton absorption pathway to the $\mathrm{A}{ }^{2} \Sigma_{\mathrm{u}}^{+}$excited state and subsequently the one-photon pathway to the same final dissociative state. In this region of the potential energy surface, the $\mathrm{H}_{2}^{+}$molecule undergoes enhanced ionization producing two protons whose kinetic energy is detected with a time-of-flight mass spectrometer.

The measured kinetic energy release shows a strong spectral modulation for all laser pulse durations (40-140 fs) and wavelengths $(800 \mathrm{~nm}, 1.2 \mu \mathrm{m}, 1.4 \mu \mathrm{m})$ that were investigated for both linear and circular polarizations. Comparable modulations were also found in $\mathrm{D}_{2}$. Since the kinetic energy distributions are centered in the region of $3-7 \mathrm{eV}$, the second ionization occurs in the region of $R \sim 5-10$ a.u. and is caused by enhanced ionization. A comparison with model calculations solving the TDSE showed that the spectral modulation arises from the interplay between electronic population dynamics and the vibrational wave packet. The total wave function $\Psi\left(\vec{r}_{\mathrm{el}}, R, t\right)$ of the system is approximated as a superposition of two electronic eigenfunctions $\phi_{\mathrm{g}}^{\mathrm{el}}\left(\vec{r}_{\mathrm{el}}, R\right)$ and $\phi_{\mathrm{u}}^{\mathrm{el}}\left(\vec{r}_{\mathrm{el}}, R\right)$ :

$$
\Psi\left(\vec{r}_{\mathrm{el}}, R, t\right)=\psi_{\mathrm{g}}(R, t) \phi_{\mathrm{g}}^{\mathrm{el}}\left(\vec{r}_{\mathrm{el}}, R\right)+\psi_{\mathrm{u}}(R, t) \phi_{\mathrm{u}}^{\mathrm{el}}\left(\vec{r}_{\mathrm{el}}, R\right)
$$

Asymptotically, $\psi_{\mathrm{u}}(R, t)$ and $\psi_{\mathrm{g}}(R, t)$ become proportional to $\exp \left(\mathrm{i} k_{\mathrm{u}} R\right)$ and $\exp \left(\mathrm{i} k_{\mathrm{g}} R\right)$, respectively, where $k_{\mathrm{u}}$ and $k_{\mathrm{g}}$ are the nuclear momenta corresponding to the one- or net-two-photon (absorption of three photons and emission of one photon) channels. The interference between these channels results in a spatial modulation of the upper state 
population, which is mapped onto the kinetic energy release of the fragments.

This experiment has revealed that the electronic population of the quasistatic upper potential energy surface of dissociating $\mathrm{H}_{2}^{+}$and $\mathrm{D}_{2}^{+}$has a strong $R$-dependent modulation, which is sampled by field ionization twice per laser period with a 360 -as $(800-\mathrm{nm})$ to 620 -as $(1400-\mathrm{nm})$ window. The experiment shows that the electronic population dynamics of a molecule can be imaged in a strong laser field. Interesting extensions include the observation of more complex electronic dynamics (e.g., ring currents) or the observation of coupled nuclear and electronic motions in polyatomic molecules.

\section{IMAGING OF MOLECULAR STRUCTURE}

Spectroscopy has been of fundamental importance in determining the structure of small molecules. However the wavelength of optical sources is just too long for a direct structural measurement. Therefore, the forefront of molecular imaging has passed to synchrotrons, which produce $10 \mathrm{keV}(\lambda \sim 1 \AA)$ light.

For typical parameters of a strong laser field (intensity $\sim 10^{14} \mathrm{~W} \mathrm{~cm}^{-2}, \lambda \leq 800 \mathrm{~nm}$ ), the recolliding electron has a de Broglie wavelength of $0.5-5 \AA$. This is the natural scale of chemical bonds and orbitals that can therefore be imaged in the recollision process. There are currently three main methods to retrieve spatial information about the investigated molecules. The first consists in measuring the spectral amplitude, phase, and polarization of the highorder harmonics emitted during the recollision. This process is repeated under different recollision angles of the electron wave packet in a way that is formally analogous to medical tomography and allows the reconstruction of molecular orbitals. The second method exploits the diffraction information contained in electrons that are elastically scattered from the molecular target. In contrast to traditional electron diffraction, LIED relies on the molecule's own electrons and therefore achieves extremely high current densities. Moreover, the recollision can be timed with attosecond precision (Section 5.2.1), which allows the observation of dynamics. The third method is electron wave packet interferometry exploiting the concepts of optical holography. An attosecond XUV pulse train generates a series of photoelectron replica that are subjected to the action of an intense IR laser field. The field induces momentum shifts of the photoelectron wave packets that interfere with each other. The experiment thereby provides information on the action of the field and the nature of the electron wave packets.

\subsection{Tomography of Molecular Orbitals}

The process of high harmonic generation, which constitutes the basis of orbital tomography, is best described in the language of interferometry (Section 3.1). The strong laser field acts on the wave function of the molecule like a beam splitter and produces a coherent superposition state (equation 8) with a dominant bound part and a small amplitude of a singly ionized state with a very broad spectrum (typically tens of electronvolts). The laser field dominates the time evolution of the continuum wave packet and causes its return to the parent molecule where it interferes with the bound part of the wave function and produces an oscillating dipole. This last step is equivalent to overlapping beams in interferometry and hence complete information about the bound and continuum parts of the wave function is encoded in the emitted radiation. By the time of recollision, the electron wave packet has reached a lateral width of $10 \AA$, which exceeds the dimension of an atom or a small molecule. Therefore, the wave packet $\left|\Psi_{\mathrm{c}}\right\rangle$ is approximated by a set of plane waves with electronic wave vector $\vec{k}$ and complex coefficients $a(k)$ as given in equation (14).

The transition dipole moment from the ionized state back into the ground state $\Psi_{\mathrm{g}}$ is then

$$
\vec{d}(\omega) \propto a[k(\omega)] \int \Psi_{\mathrm{g}}(\vec{r}) \vec{r} \exp [\mathrm{i} k(\omega) x] \mathrm{d} \vec{r}
$$

which determines the spectral amplitude $I(\omega) \propto \omega^{4}|d(\omega)|^{2}$ and phase $\alpha(\omega)=\arg [d(\omega)]$ of the emitted harmonic radiation. If the continuum wave packet can be accurately characterized, i.e., if $a[k(\omega)]$ is known, measuring the harmonic spectrum is equivalent to experimentally determining the spatial Fourier transform of $\vec{r} \Psi_{\mathrm{g}}(\vec{r})$.

The determination of $a[k(\omega)]$ is one of the critical steps. In Itatani et al. (2004), which describes the tomography of $\mathrm{N}_{2}, a[k(\omega)]$ was determined in $\mathrm{Ar}$, which has a similar ionization energy and polarizability. The intensity-dependent ionization rates for $\mathrm{Ar}$ and $\mathrm{N}_{2}$ under different orientations are similar and therefore the ionization step of harmonic generation is comparable in the two systems. Moreover, tunneling ionization occurs through a spatially narrow barrier that acts as a filter on the electron wave function and removes much of the orbital structure from the continuum wave packet. Therefore, the essential characteristics of the continuum wave packet, in particular its time and frequency structure, are comparable. This means that $a[k(\omega)]$ can be determined using equation (19) and the calculated orbital wave function of the reference atom.

The application of equation (19) to a particular system depends on the symmetry of the involved orbitals. Owing to its exponential dependence on ionization energy 
(equation 2), tunneling ionization is usually capable of selecting individual orbitals on the basis of their binding energy. However, the ionization rate also strongly depends on the spatial structure of the orbitals. In the rare gas atoms, calculations (Taieb et al. 2001) and experiments (Young et al. 2006, Loh et al. 2007) show that the p orbital aligned along the field direction is selectively ionized. In diatomic molecules, $\sigma$ orbitals have a weaker dependence of ionization rate on the angle relative to the molecular axis than $\pi$ orbitals that have a highly anisotropic tunnel ionization rate. In $\mathrm{N}_{2}$, the orbital with lowest binding energy is of $\sigma_{\mathrm{g}}$ symmetry and the ionization rate varies slowly with angle (Pavičić et al. 2007). Its high symmetry permits the complete characterization of the transition dipole by variation of the angle $\theta$ between the molecular axis and the direction of recollision. Experimentally, this is achieved by aligning the molecules in space (Itatani et al. 2005) before harmonic generation and varying the angle of alignment relative to the polarization direction of the strong laser field.

Another essential quantity is the phase $\alpha(\omega)$ of the dipole moment. It can be measured experimentally by the RABBITT technique (Mairesse et al. 2003). In $\mathrm{N}_{2}$ the phase of the dipole moment for emission polarized along the molecular axis seems to undergo a sudden variation at medium photon energies $(39 \mathrm{eV})$, whereas no such variation is observed in the direction perpendicular to the molecular axis (Levesque et al. 2007). This property has been interpreted as the signature of the nodal structure of the orbital and is an essential ingredient in the tomographic procedure. Knowing amplitudes and phases of the transition moment $\vec{d}(\omega)$ under a set of angles $\theta$, the three-dimensional structure of $\Psi_{\mathrm{g}}$ is reconstructed from the experimental data by the application of the modified Fourier slice theorem, also known as the Radon transformation in medical tomography (Kak and Slaney 2001).

In the discussion of molecular orbital tomography, we have ignored multielectron effects so far. In most molecules, the exchange interaction and electron correlation play an important role. Recent progress in the theory of tomographic imaging has shown that the inclusion of these effects modifies the interpretation of the extracted orbital wave function (Patchkovskii et al. 2006, 2007, Santra and Gordon 2006). Rather than the highest occupied molecular orbital wave function, the tomographic procedure provides the Dyson orbital, which is defined as the overlap between an $N$ electron molecular wave function and the $N-1$ electron wave function of the corresponding cation. Figure 9(a) shows the molecular orbital reconstructed from the experimental data, the calculated Hartree-Fock $3 \sigma_{\mathrm{g}}$ orbital of $\mathrm{N}_{2}$ (Figure 9b), and the theoretically predicted result using the multielectron theory for high harmonic generation (Figure 9c).
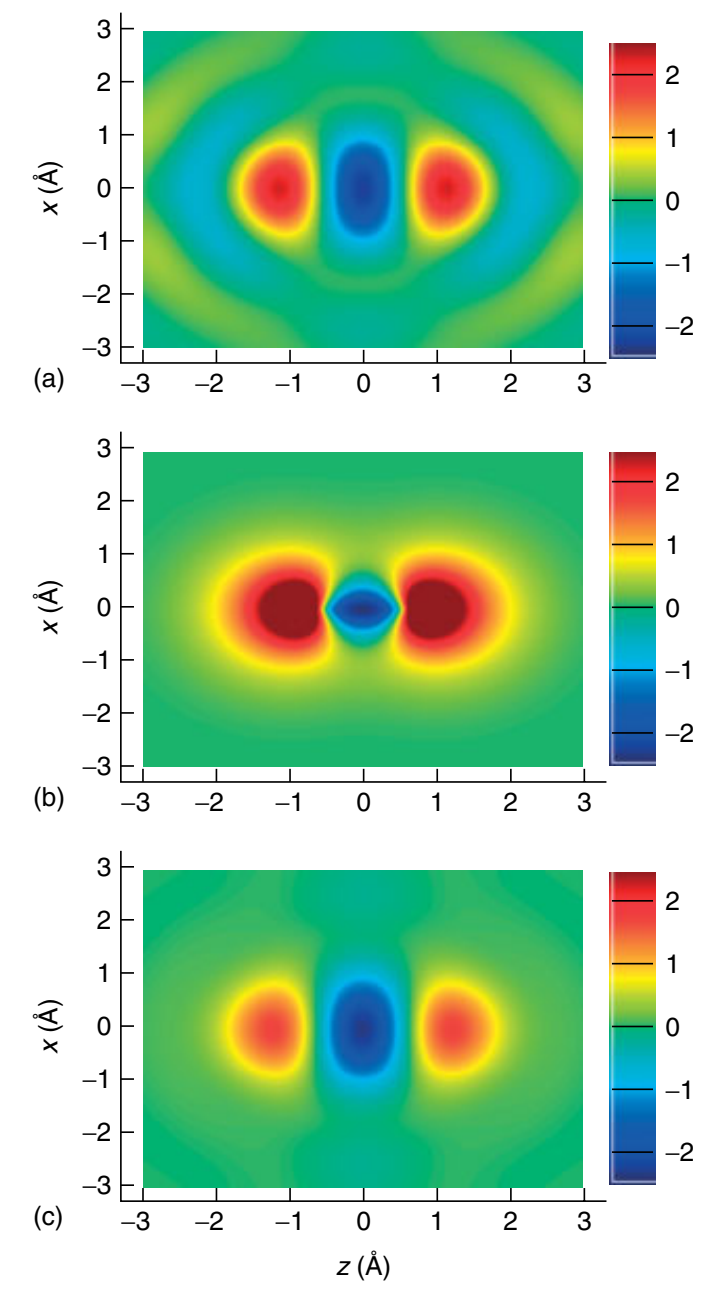

Figure 9 Two-dimensional projections of the orbital wave functions of $\mathrm{N}_{2}$. (a) This shows the orbital obtained from the tomographic reconstruction using experimental high harmonic spectra of nitrogen molecules. (b) This represents the calculated Hartree-Fock $3 \sigma_{\mathrm{g}}$ highest occupied molecular orbital of $\mathrm{N}_{2}$. (c) This shows the predicted measurement using the multielectron theory for high harmonic generation. [Reproduced from Patchkovskii et al. (2007) with permission.]

The unique characteristic of tomographic imaging using high harmonic generation is its ultrafast timescale. The elementary steps of the process have a duration of less than a laser cycle or less than $2.7 \mathrm{fs}$ at $800 \mathrm{~nm}$, which is much faster than the motion of any but the lightest nuclei. Consequently, tomographic imaging can be applied to observe the modification of orbitals during a chemical reaction with the potential of verifying the most fundamental concepts of chemistry.

\subsection{Laser-induced Electron Diffraction}

The recolliding electron wave packet is characterized by unique properties. Seen from the ion, the electron current 


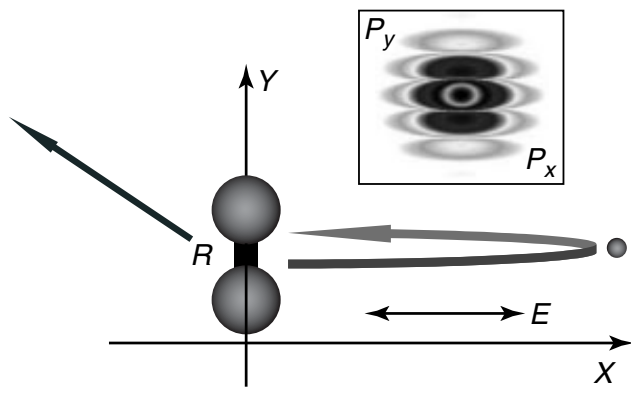

Figure 10 Illustration of laser-induced electron diffraction. The inset sketches the ground-state wave function of a diatomic molecule in momentum space with the grayscale coding the probability density. [Reproduced from Spanner et al. 2004 with permission.]

density amounts to more than $10^{10} \mathrm{~A} \mathrm{~cm}^{-2}$ and is concentrated in a small fraction of the laser cycle. These properties suggest that LIED may have advantages over conventional methods of ultrafast electron diffraction (Cao et al. 1999).

Molecular diffraction imaging using interference patterns in strong-field ionization was originally proposed in Zuo et al. (1996), where the angular distribution of direct electrons from stretched $\mathrm{H}_{2}^{+}$was found to contain the signatures of two-center interference. The electron waves originating from the two centers produce an interference pattern that varies with the internuclear distance. The structure of the PAD was shown to be characteristic of the internuclear separation. The interference phenomenon is even stronger when the electrons are forced to recollide with the parent ion and are scattered elastically by the molecular core. The rescattered electrons reach a much higher cutoff energy $\left(10 U_{\mathrm{p}}\right)$ than the direct electrons $\left(2 U_{\mathrm{p}}\right)$ and can therefore be distinguished experimentally. Interference in elastic rescattering of the high-energy ATI electrons has been observed very recently (Meckel et al. 2008) and has been analyzed theoretically in Spanner et al. (2004), which details the interpretation of the diffraction patterns.

In the initial tunnel ionization step, the lateral velocity distribution of the created continuum electron wave packet is determined by the momentum space representation of the ionized orbital multiplied by a Gaussian tunneling filter (Figure 10):

$$
\Psi\left(v_{\perp}, t_{0}\right) \propto\left\langle v_{\perp} \mid \Psi_{\mathrm{g}}\right\rangle \exp \left(-\frac{v^{2}}{2} \tau_{\mathrm{T}}\left(t_{0}\right)\right)
$$

where $\tau_{\mathrm{T}}=\sqrt{2 I_{\mathrm{p}}} / E\left(t_{0}\right)$ is the tunneling time and $t_{0}$ is the moment of ionization. The electron wave packet is subsequently accelerated away from the ion core and then back to it where it is elastically scattered. Interference structures appear in the spectrum and angular distribution of the rescattered electrons. The electron momentum distribution is influenced by several effects:

(i) The component of the recolliding wave packet that has started with $v_{\perp}=0$ but has gained a lateral velocity component $v_{\perp}$ during recollision will interfere with the wave that has started with nonzero $v_{\perp}$ and has missed the ion. This holographic interference is common to atoms and molecules.

(ii) After rescattering, the electron is still subject to the strong laser field that modifies its longitudinal velocity while the transversal velocity is unaffected. The angleresolved spectrum for a given final energy, therefore, contains contributions from scattering at different recollision energies.

(iii) The contribution of electron trajectories corresponding to a different recollision energy and recollision time to the same final vector velocity results in interference that is exclusively caused by the laser field.

The calculated two-dimensional electron spectra for a model diatomic molecule are shown in Figure 11. In the case of a near-single-cycle pulse (Figure 11a), the electron momenta are mostly located within a circle that is shifted from the origin as a consequence of the momentum shift due to the laser field. The concentric ring structure that is visible close to the outer circle results from the interference of short and long trajectories. Figure 11(b) represents the amplitude of the momentum distribution along the red circle shown in Figure 11(a). The dominant triple-peaked structure close to zero momentum results from holographic interference and its width characterizes the initial momentum distribution of the ionized wave packet. The deep amplitude modulation of the momentum distribution is the result of interferences due to the molecular structure. Figure 11(c) illustrates the case of a realistic 5-fs laser pulse with zero CEP. In this case, the momentum distribution is concentrated within two circles of different radii corresponding to the dominant recollision events. The amplitude of the momentum distribution is plotted in Figure 11(d) and is found to exhibit similar interference patterns.

The results of three-dimensional TDSE simulations of $\mathrm{H}_{2}^{+}$show that the internuclear distance can directly be retrieved from LIED (Yurchenko et al. 2004). Yurchenko et al. (2004) study a model diatomic molecule aligned perpendicular to the direction of the laser polarization. A clear diffraction pattern can be retrieved from electron momentum distributions by taking the ratio of the distribution along the molecular axis to the distribution along the perpendicular direction. The latter serves as a reference that characterizes the shape of the momentum distribution induced by the initial orbital and the action of the laser field. The periodicity in momentum space is found to be 

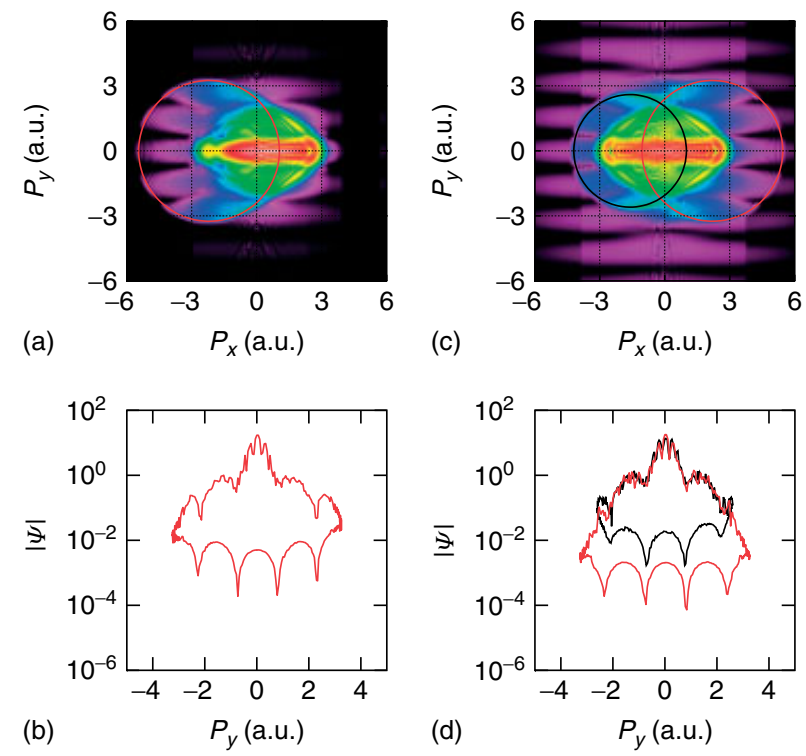

Figure 11 Electron spectrum $\left|\Psi\left(p_{x}, p_{y}\right)\right|$ of the electrons emitted from a diatomic molecule exposed to a 1.25 cycle pulse of constant amplitude $\left(I \approx 6.9 \times 10^{14} \mathrm{~W} \mathrm{~cm}^{-2}, \lambda=800 \mathrm{~nm}\right.$, (a) $)$ and a 5 -fs pulse with a $\cos ^{2}$ envelope function (c). (b) and (d) These represent spectral cuts through the functions shown in (a) and (c), respectively, for a fixed moment of recollision $(\omega t \approx 4.4$ rad). [Reproduced from Spanner et al. (2004) with permission.]

$\Delta p \approx 2 \pi / R$, which provides a measure of the internuclear separation $R$.

As outlined in the preceding section, the key advantage of the present strong-field variant over traditional methods of electron diffraction is its high temporal resolution. Since the diffraction experiment is completed in less than one laser cycle, ultrafast chemical transformations can be imaged in real time with unprecedented spatial resolution.

\subsection{Holography}

The interference of continuum electron wave packets also lies at the basis of another technique that has the potential to allow molecular imaging: attosecond electron wave packet interferometry (Remetter et al. 2006). An attosecond pulse train is used to ionize the target system and produces a series of photoelectron wave packets corresponding to the photon energies of the harmonics that constitute the pulse train. A synchronized IR field induces a momentum shear between consecutive electron wave packets that are thereby forced to interfere with each other. The interferogram contains information about the relative phases of the wave packets. The method has the potential of achieving complete (amplitude and phase) characterization of the continuum electron wave packets and may remove the need for a reference system to characterize the continuum wave packet (Section 6.1).
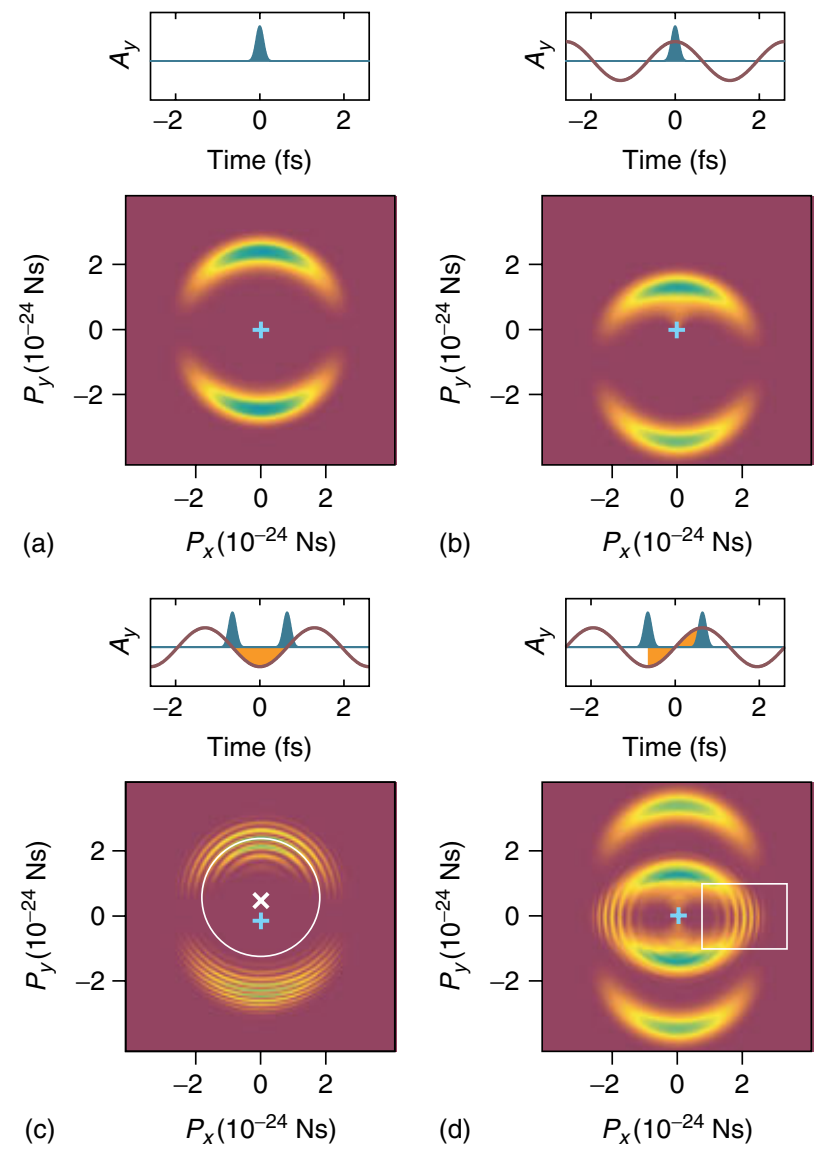

Figure 12 The results of calculations carried out by solving the time-dependent Schrödinger equation in helium within the single active electron approximation. (a) The momentum distribution of an electron ionized by a single 180 as $46 \mathrm{eV}$ pulse. (b) The distribution is displaced because the ionization occurs in the presence of an IR laser field that is linearly polarized along the $y$ axis. (c) and (d) the photoionization is due to a sequence of two attosecond pulses separated by half the IR laser cycle. (d) The ionization occurs at a maximum or minimum of $A(\tau)$, and there is a momentum shear between the wave packets that affects the interference pattern in the region of overlap. [Reproduced from Remetter et al. (2006) with permission.]

In optical interferometry, the phase $\phi(x)$ of an electric field $E(x)$ can be determined by splitting the field in two replicas and adding a "shear" $\delta x$ to one of the replicas. Assuming comparable amplitudes of the fields in the region of overlap the interferogram is given by $|E(x)+E(x+\delta x)|^{2} \propto 1+\cos [\phi(x)-\phi(x+\delta x)]$, which allows the determination of the phase $\phi(x)$. The principles of attosecond electron wave packet interferometry are related to those of the attosecond streak camera (Section 4.2) and are illustrated in Figure 12. When a single attosecond XUV pulse photoionizes a He atom, the photoelectron has a p-type momentum distribution with an average radius corresponding to the average photoelectron momentum and the width reflecting the bandwidth of 
the attosecond pulse. In the presence of a strong IR field, the momentum distribution is shifted by an amount that depends on the phase of the IR field. In Figure 12(c) and (d), the ionization is induced by a pair of attosecond pulses separated by half the period of the IR field. We consider two limiting cases. If the ionization takes place when the vector potential of the IR field crosses zero (c), the final momentum of the electron wave packet is not affected by the IR field. However, the wave packets spend a different amount of time in the IR field and therefore accumulate a different phase that leads to the observed interference fringes. When ionization takes place at the maxima of the vector potential, the electron wave packets undergo a velocity change that has opposite signs for consecutive pulses. In momentum space, the corresponding distribution lies on circles centered on $p_{y}= \pm A_{L}(\tau) / \pi$ where $p_{y}$ is the electron momentum along the direction of the laser polarization and $A_{L}(\tau)$ is the magnitude of the laser vector potential at the moment of ionization. Hence, the two contributions interfere in the region of overlap providing an interferogram of the electron wave packets in momentum space.

The experimental realization of the scheme described above was achieved by generating an attosecond pulse train in argon and overlapping it with the residual fundamental field at an intensity of $10^{13} \mathrm{~W} \mathrm{~cm}^{-2}$ in the detection region of a velocity-map-imaging spectrometer. The photoelectron images recorded for two different delays $\left(A_{L}(\tau)=0\right.$ and $\left.A_{L}(\tau)=A_{\max }\right)$ are shown in Figure 13. The observed images are more complex than the simulated ones because ionization of argon generates $\mathrm{s}$ and $\mathrm{d}$ waves (instead of $\mathrm{p}$ waves in $\mathrm{He}$ ) and the ionization is induced by a pulse train that consists of several harmonic frequency components. Figure 13(a) corresponds to no net shearing of the electron wave packets and its interference structure allows an accurate determination of the intensity of the IR field. Figure 13(b) corresponds to maximal shearing and reveals the interference patterns predicted by the simulations of Figure 12(d) in the region highlighted by the white frame. The comparison with simulations (not shown) reveals that the interference pattern is highly sensitive to the symmetry of the initial wave function.

Generally, this interferometric method should permit the characterization of the phase structure of the initial continuum wave packet in the region where the final momenta overlap. The technique can be generalized to twodimensional phase reconstruction by using a momentum shear in two perpendicular directions. Extension of the measurements to small momentum shears provides a larger region of overlap, whereas a large momentum shear will allow to probe the phase difference of different parts of the wave packet, which may be an accurate alternative in determining the chirp of electron wave packets. Moreover, varying the polarization between the XUV and IR fields will
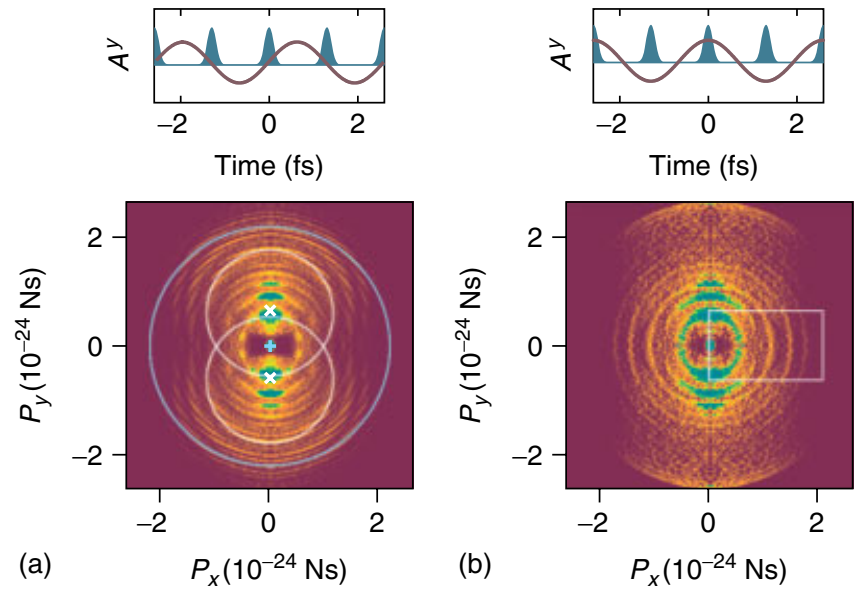

Figure 13 Experimental data at two different relative delays between the attosecond pulses and the IR laser field. The attosecond pulses coincide with the zeros of the vector potential in (a) and with its maxima and minima in (b). In (a), the fringes resulting from the interference of consecutive electron wave packets are circles centered on the $p_{y}$ axis, as shown by the small circles centered on the white crosses. Those owing to interferences between electron wave packets separated by one IR cycle are circles centered at the origin, as shown by the large circle centered on the blue plus sign. In (b), the most remarkable effect is the pronounced interference structure crossing the $p_{x}$ axis, as shown inside the white rectangle. [Reproduced from Remetter et al. 2006 with permission.]

enable the characterization of a larger region of momentum space. The method may thus open new opportunities in the characterization of bound and continuum electron wave functions and their evolution on an attosecond timescale.

\section{FUTURE PROSPECTS}

We have outlined the main thrusts of attosecond science, with an emphasis on the aspects that could have the greatest impact on spectroscopy. So far, only proof-of-principle experiments have been performed, but these experiments hold significant promise.

Attosecond spectroscopy in general and HHG in particular are strongly related to photoionization and photoelectron spectroscopy (see Pratt 2011: High-resolution Valenceshell Photoionization, Merkt et al. 2011: High-resolution Photoelectron Spectroscopy, this handbook, respectively). High-harmonic generation allows the measurement of photorecombination cross-sections over energy intervals of typically $10-100 \mathrm{eV}$. The measured cross-section is differential in space (angle-resolved) and in electronic state (when only one orbital ionizes). We have shown how attosecond spectroscopy can be used to study time-resolved processes in molecular cations. This approach will complement cation spectroscopy (see Willitsch 2011: Experimental Methods 
in Cation Spectroscopy, this handbook) with the advantage that the molecule is initially in its neutral ground state. The technology underlying attosecond pulse generation which is further described in Gallmann and Keller 2011: Femtosecond and Attosecond Light Sources and Techniques for Spectroscopy (this handbook), will also enhance XUV precision spectroscopy (see Eikema and Ubachs 2011: Precision Laser Spectroscopy in the Extreme Ultraviolet, this handbook) through the realization of highharmonic frequency combs (Gohle et al. 2002, Jones et al. 2005).

We have shown how light can control collisions. This means that collisions can be timed with respect to another light-driven event, such as photoexcitation. We have also shown that laser-driven collisions can be used to image orbitals or the position of atoms in a molecule. Putting these two together, the imaging of chemical dynamics is possible.

We have shown that attosecond XUV pulses can be used to excite intra-atomic dynamics in krypton and xenon. The strong, phased laser field provides two ways to follow the dynamics: (i) by using the strong field as a gate to allow electron tunneling within only a short time window and (ii) by using the laser field to streak electrons. This same process can be extended to solids (Cavalieri et al. 2007).

Attosecond XUV pulses are not the only way to excite attosecond dynamics. Collision physics has been exciting attosecond processes for decades. However, it has lacked a means of measuring this dynamics. Tunneling and streaking are two ways. Both are being used in collision experiments. In fact, it seems clear that optical quality measurement methods, such as gating, can be extended to recollision experiments. This will unlock many new phenomena for study.

One of the most revolutionary aspects of the new technology is how correlation is built into it. Correlation always enhanced measurements. We have summarized how it is used to measure the early motion of an $\mathrm{H}_{2}^{+}$wave packet. It will also be possible to measure electron dynamics that is launched by tunneling. It can be used in space or in time.

It is important to comment on the potential of molecular imaging. Because it is a correlated measurement-often one molecule at a time-it is possible to select molecules (for example, isomers or enantiomers) for imaging. Only those need to be imaged. Similarly during photodissociation, only one pathway needs to be imaged.

At present, the relatively low energy of the recollision electrons appears to limit their use to relatively small molecules. However, there is no reason why IR-driven lasers cannot be used, allowing the energies to approach or exceed a kilo electronvolt.

Finally, multiphoton physics has been essential in reaching the attosecond frontier. It is the inherently multiphoton nature of the interaction that allows the laser period to be subdivided. Electron recollision is only one specific multiphoton process. Tunneling is another. There will be still others. All will open new forms of measurement. They will broaden the range of phenomena to be measured and controlled.

\section{ACKNOWLEDGMENTS}

One of the authors (H. J. W.) acknowledges support from the Swiss National Science Foundation.

\section{ABBREVIATIONS AND ACRONYMS}

$\begin{array}{ll}\text { CEP } & \text { carrier envelope phase } \\ \text { HHG } & \text { High-harmonic generation } \\ \text { IR } & \text { infrared } \\ \text { LIED } & \text { laser-induced electron diffraction } \\ \text { MFPAD } & \text { Molecular frame PAD } \\ \text { PAD } & \text { photoelectron angular distribution } \\ \text { SFA } & \text { strong-field approximation } \\ \text { TDSE } & \text { time-dependent Schrödinger equation } \\ \text { XUV } & \text { extreme-ultraviolet }\end{array}$

\section{REFERENCES}

Ammosov, M.V., Delone, N.B., and Krainov, V.P. (1986) Tunnel ionization of complex atoms and of atomic ions in an alternating electromagnetic field. Soviet Physics JETP, 91, 2008.

Aseyev, S.A., Ni, Y., Frasinski, L.J., Muller, H.G., and Vrakking, M.J.J. (2003) Attosecond angle-resolved photoelectron spectroscopy. Physical Review Letters, 91, 223902.

Bai, C. (2000) Scanning Tunneling Microscopy and Its Application, Springer.

Baker, S., Robinson, J.S., Haworth, C.A., Teng, H., Smith, R.A., Chirila, C.C., Lein, M., Tisch, J.W.G., and Marangos, J.P. (2006) Probing proton dynamics in molecules on an attosecond time scale. Science, 312, 424.

Baltuska, A., Udem, T., Uiberacker, M., Hentschel, M., Goulielmakis, E., Gohle, C., Holzwarth, R., Yakovlev, V.S., Scrinzi, A., Hänsch, T.W. et al. (2003) Attosecond control of electronic processes by intense light fields. Nature, 421, 611-615.

Cao, J., Ihee, H., and Zewail, A.H. (1999) Ultrafast electron diffraction and direct observation of transient structures in a chemical reaction. Proceedings of the National Academy of Sciences, 96, 338-342.

Cavalieri, A.L., Muller, N., Uphues, T., Yakovlev, V.S., Baltuska, A., Horvath, B., Schmidt, B., Blumel, L., Holzwarth, R., Hendel, S. et al. (2007) Attosecond spectroscopy in condensed matter. Nature, 449(7165), 1029.

Chu, S. (2002) Cold atoms and quantum control. Nature, 416, 206-210. 
Corkum, P.B. (1993) Plasma perspective on strong-field multiphoton ionization. Physical Review Letters, 71, 1994-1997.

Corkum, P.B. (1994) Subfemtosecond pulses. Optics Letters, 19, 1870.

Corkum, P.B. and Krausz, F. (2007) Attosecond science. Nature Physics, 3, 381-387.

Cundiff, S.T., Ye, J., and Hall, J.L. (2001) Optical frequency synthesis based on mode-locked lasers. Review of Scientific Instruments, 72, 3749.

Dietrich, P., Burnett, N.H., Ivanov, M., and Corkum, P.B. (1994) High-harmonic generation and correlated two-electron multiphoton ionization with elliptically polarized light. Physical Review A, 50, R3585-R3588.

Drescher, M., Hentschel, M., Kienberger, R., Uiberacker, M., Yakolev, V., Scrinzi, A., Westerwalbesloh, T., Kleineberg, U., and Krausz, F. (2002) Time-resolved atomic inner-shell spectroscopy. Nature, 419, 803-807.

Dudovich, N., Smirnova, O., Levesque, J., Ivanov, M., Villeneuve, D.M., and Corkum, P.B. (2006) Measuring and controlling the birth of attosecond pulses. Nature Physics, 2,781 .

Eikema, K.S.E. and Ubachs, W. (2011) Precision laser spectroscopy in the extreme ultraviolet, in Handbook of Highresolution Spectroscopy, Quack, M. and Merkt, F. (eds), John Wiley \& Sons, Ltd., Chichester, UK.

Ferray, M., L'Huillier, A., Li, X.F., Lompre, L.A., Mainfray, G., and Manus, C. (1988) Multiple-harmonic conversion of $1064 \mathrm{~nm}$ radiation in rare gases. Journal of Physics B, 21, L31.

Fischer, M., Kolachevsky, N., Zimmermann, M., Holzwarth, R., Udem, T., Hänsch, T.W., Abgrall, M., Grünert, J., Maksimovic, I., Bize, S. et al. (2004) New limits on the drift of fundamental constants from laboratory measurements. Physical Review Letters, 92(23), 230802.

Gallmann, L. and Keller, U. (2011) Femtosecond and attosecond light sources and techniques for spectroscopy, in Handbook of High-resolution Spectroscopy, Quack, M. and Merkt, F. (eds), John Wiley \& Sons, Ltd., Chichester, UK.

Gamow, G. (1929) Successive alpha-transformations. Nature, 123, 606.

Gessner, O., Lee, A., Shaffer, J., Reisler, H., Levchenko, S., Krylov, A., Underwood, J.G., Shi, H., East, A., Wardlaw, D. et al. (2006) Femtosecond multi-dimensional imaging of a molecular dissociation. Science, 311, 219.

Gohle, C., Udem, Th., Herrmann, M., Rauschenberger, J., Holzwarth, R., Schuessler, H.A., Krausz, F., and Hänsch, T.W. (2002) A frequency comb in the extreme ultraviolet. Nature, 416, 233-237.

Goulielmakis, E., Schultze, M., Hofstetter, M., Yakovlev, V.S., Gagnon, J., Uiberacker, M., Aquila, A.L., Gullikson, E.M., Attwood, D.T., Kienberger, R. et al. (2008) Single-cycle nonlinear optics. Science, 320(5883), 1614-1617.

Herzberg, G. (1989) Molecular Spectra and Molecular Structure, Volume I, Spectra of Diatomic Molecules, 2nd edition, Krieger Publishing Company, Malabar.

Herzberg, G. (1991a) Molecular Spectra and Molecular Structure, Volume II, Infrared and Raman Spectra of Polyatomic Molecules, Krieger Publishing Company, Malabar.
Herzberg, G. (1991b) Molecular Spectra and Molecular Structure, Volume III, Electronic Spectra and Electronic Structure of Polyatomic Molecules, 2nd edition, Krieger Publishing Company, Malabar.

Hu, S.X. and Collins, L.A. (2006) Attosecond pump probe: exploring ultrafast electron motion inside an atom. Physical Review Letters, 96, 073004.

Huber, K.P. and Herzberg, G. (1979) Molecular Spectra and Molecular Structure, Volume IV, Constants of Diatomic Molecules, Van Nostrand Reinhold Company, New York.

Hund, F. (1927) Zur deutung der molekelspektren. III. Zeitschrift für Physik, 43(11-12), 805-826.

Itatani, J., Levesque, J., Zeidler, D., Niikura, H., Pépin, H., Kieffer, J.C., Corkum, P.B., and Villeneuve, D.M. (2004) Tomographic imaging of molecular orbitals. Nature, 432, 867.

Itatani, J., Quéré, F., Yudin, G.L., Ivanov, M.Y., Krausz, F., and Corkum, P.B. (2002) Attosecond streak camera. Physical Review Letters, 88, 173903.

Itatani, J., Zeidler, D., Levesque, J., Spanner, M., Villeneuve, D.M., and Corkum, P.B. (2005) Controlling high harmonic generation with molecular wave packets. Physical Review Letters, 94, 123902.

Jones, R.J., Moll, K.D., Thorpe, M.J., and Ye, J. (2005) Phasecoherent frequency combs in the vacuum ultraviolet via high-harmonic generation inside a femtosecond enhancement cavity. Physical Review Letters, 94, 193901.

Kak, A.C. and Slaney, M. (2001) Principles of Computerized Tomographic Imaging, Society for Industrial and Applied Mathematics, New York.

Kienberger, R., Goulielmakis, E., Uiberacker, M., Baltuska, A., Yakovlev, V., Bammer, F., Scrinzi, A., Westerwalbesloh, T., Kleineberg, U., Heinzmann, U. et al. (2003) Atomic transient recorder. Nature, 427, 817-821.

Kimura, K., Katsumata, S., Achiba, Y., Yamazaki, T., and Iwata, S. (1981) Handbook of HeI Photoelectron Spectra of Fundamental Organic Molecules : Ionization Energies, ab initio Assignments, and Valence Electronic Structure for 200 Molecules, Japan scientific Societies Press, Tokyo.

Kovacev, M., Mairesse, Y., Priori, E., and Merdji, H. (2003) Temporal confinement of the harmonic emission through polarization gating. European Physical Journal D, 26, 79-82.

Krainov, V. (1997) Ionization rates and energy and angular distributions at the barrier-suppression ionization of complex atoms and atomic ions. Journal of the Optical Society of America B, 14, 425.

Kuleff, A.I., Breidbach, J., and Cederbaum, L.S. (2005) Multielectron wave-packet propagation: General theory and application. Journal of Chemical Physics, 123, 044111.

Lein, M. (2005) Attosecond probing of vibrational dynamics with high-harmonic generation. Physical Review Letters, 94, 053004.

Levesque, J., Mairesse, Y., Dudovich, N., Pépin, H., Kieffer, J.C., Corkum, P.B., and Villeneuve, D.M. (2007) Polarization state of high-order harmonic emission from aligned molecules. Physical Review Letters, 99, 243001.

Lewenstein, M., Balcou, P., Ivanov, M.Y., L'Huillier, A., and Corkum, P. (1994) Theory of high-harmonic generation by lowfrequency laser fields. Physical Review A, 49, 2117. 
Lezius, M., Blanchet, V., Ivanov, M., and Stolow, A. (2002) Polyatomic molecules in strong laser fields: nonadiabatic multielectron dynamics. Journal of Chemical Physics, 117, 1575.

Lezius, M., Blanchet, V., Rayner, D., Villeneuve, D.M., Stolow, A., and Ivanov, M. (2001) Non-adiabatic multi-electron dynamics in strong field molecular ionization. Physical Review Letters, 86, 51 .

Loh, Z.H., Kahil, M., Correa, R.E., Santra, R., Buth, C., and Leone, S.R. (2007) Quantum state-resolved probing of strongfield-ionized xenon atoms using femtosecond high-order harmonic transient absorption spectroscopy. Physical Review Letters, 98(143601), 143601.

Mairesse, Y., de Bohan, A., Frasinski, L.J., Merdji, H., Dinu, L.C., Monchicourt, P., Breger, P., Kovacev, M., Taieb, R., Carré, B. et al. (2003) Attosecond synchronization of highharmonic soft X-rays. Science, 302, 1540.

McPherson, A., Gibson, G., Jara, H., Johann, U., Luk, T.S., McIntyre, I.A., Boyer, K., and Rhodes, C.K. (1987) Studies of multiphoton production of vacuum-ultraviolet radiation in the rare gases. Journal of the Optical Society of America B, 4, 595.

Meckel, M., Comtois, D., Zeidler, D., Staudte, A., Pavičić, D., Bandulet, H.C., $\quad$ Pépin, H., $\quad$ Kieffer, J.C., $\quad$ Dörner, R., Villeneuve, D.M. et al. (2008) Laser induced electron tunnelling and diffraction. Science, 320, 1478.

Merkt, F. and Quack, M. (2011) Molecular quantum mechanics and molecular spectra, molecular symmetry, and interaction of matter with radiation, in Handbook of High-resolution Spectroscopy, Quack, M. and Merkt, F. (eds), John Wiley \& Sons, Ltd., Chichester, UK.

Merkt, F., Willitsch, S., and Hollenstein, U. (2011) High-resolution photoelectron spectroscopy, in Handbook of High-resolution Spectroscopy, Quack, M. and Merkt, F. (eds), John Wiley \& Sons, Ltd., Chichester, UK.

Morishita, T., Watanabe, S., and Lin, C.D. (2007) Attosecond light pulses for probing two-electron dynamics of helium in the time domain. Physical Review Letters, 98, 083003.

Mukamel, S. (1999) Principles of Nonlinear Optical Spectroscopy, Oxford University Press, USA.

Niikura, H., Légaré, F., Hasbani, R., Bandrauk, A.D., Ivanov, M.Y., Villeneuve, D.M., and Corkum, P.B. (2002) Sub-lasercycle electron pulses for probing molecular dynamics. Nature, 417, 917.

Niikura, H., Légaré, F., Hasbani, R., Ivanov, M., Villeneuve, D.M., and Corkum, P.B. (2003) Probing molecular dynamics with attosecond resolution using correlated wave packet pairs. Nature, 421, 826-829.

Patchkovskii, S., Zhao, Z., Brabec, T., and Villeneuve, D.M. (2006) High harmonic generation and molecular orbital tomography in multielectron systems: beyond the single active electron approximation. Physical Review Letters, 97(123003), 123003.

Patchkovskii, S., Zhao, Z., Brabec, T., and Villeneuve, D.M. (2007) High harmonic generation and molecular orbital tomography in multielectron systems. Journal of Chemical Physics, 126, 114306.

Paul, P.M., Toma, E.S., Breger, P., Mullot, G., Augé, F., Balcou, P., Muller, H.G., and Agostini, P. (2001) Observation of a train of attosecond pulses from high harmonic generation. Science, 292, 1689.
Paulus, G.G., Nicklich, W., Xu, H., Lambropoulos, P., and Walther, H. (1994) Plateau in above threshold ionization spectra. Physical Review Letters, 72, 2851-2854.

Pavičić, D., Lee, K.F., Rayner, D.M., Corkum, P.B., and Villeneuve, D.M. (2007) Direct measurement of the angular dependence of ionization for $\mathrm{N}_{2}, \mathrm{O}_{2}$, and $\mathrm{CO}_{2}$ in intense laser fields. Physical Review Letters, 98, 243001.

Perelemov, A., Popov, V., and Terent'ev, M. (1966) Ionization of atoms in an alternating electric field: II. Soviet Physics JETP, 23, 924.

Pratt, S.T. (2011) High-resolution valence-shell photoionization, in Handbook of High-resolution Spectroscopy, Quack, M. and Merkt, F. (eds), John Wiley \& Sons, Ltd., Chichester, UK.

Quack, M. (2011) Fundamental symmetries and symmetry violations from high-resolution spectroscopy, in Handbook of Highresolution Spectroscopy, Quack, M. and Merkt, F. (eds), John Wiley \& Sons, Ltd., Chichester, UK.

Remacle, F. and Levine, R.D. (2006) An electronic time scale in chemistry. Proceeding of the National Academy of Sciences USA, 103, 6793-6798.

Remetter, T., Johnsson, P., Mauritsson, J., Varjú, K., Ni, Y., Lépine, F., Gustafsson, E., Kling, M., Khan, J., López-Martens, R. et al. (2006) Attosecond electron wave packet interferometry. Nature Physics, 2, 323-326.

Sansone, G., Benedetti, E., Calegari, F., Vozzi, C., Avaldi, L., Flammini, R., Poletto, L., Villoresi, P., Altucci, C., Velotta, R. et al. (2006) Isolated single-cycle attosecond pulses. Science, 314, 443.

Santra, R. and Gordon, A. (2006) Three-step model for highharmonic generation in many-electron systems. Physical Review Letters, 96, 073906.

Schweigert, I.V. and Mukamel, S. (2007) Coherent ultrafast corehole correlation spectroscopy: X-ray analogues of multidimensional NMR. Physical Review Letters, 99, 163001.

Smits, M., de Lange, C., Stolow, A., and Rayner, D. (2004) Dynamic polarization in the strong field ionization of small metal clusters. Physical Review Letters, 93, 203402.

Spanner, M., Simova, O., Corkum, P.B., and Ivanov, M.Y. (2004) Reading diffraction images in strong field ionization of diatomic molecules. Journal of Physics B, 37, L243-L250.

Staudte, A., Pavičić, D., Chelkowski, S., Zeidler, D., Meckel, M., Niikura, H., Schöffler, M., Schössler, S., Ulrich, B., Rajeev, P.P. et al. (2007) Attosecond strobing of two-surface population dynamics in dissociating $\mathrm{H}_{2}^{+}$. Physical Review Letters, 98, 073003.

Taieb, R., Véniard, V., and Maquet, A. (2001) Photoelectron spectra from multiple ionization of atoms in ultra-intense laser pulses. Physical Review Letters, 87, 053002.

Tong, X.M., Zhao, Z.X., and Lin, C.D. (2002) Theory of molecular tunneling ionization. Physical Review A, 66, 033402.

Udem, T., Huber, A., Gross, B., Reichert, J., Prevedelli, M., Weitz, M., and Hänsch, T.W. (1997) Phase-coherent measurement of the hydrogen $1 \mathrm{~S}-2 \mathrm{~S}$ transition frequency with an optical frequency interval divider chain. Physical Review Letters, 79(14), 2646-2649.

Uiberacker, M., $\quad$ Uphues, T., $\quad$ Schultze, M., Verhoef, A.J., Yakovlev, V., Kling, M.F., Rauschenberger, J., Kabachnik, 
N.M., Schröder, H., Lezius, M. et al. (2007) Attosecond realtime observation of electron tunnelling in atoms. Nature, $\mathbf{4 4 6}$ 627-632.

Ullrich, J., Moshammer, R., Dorn, A., Dörner, R., Schmidt, L., and Schmidt-Böcking, H. (2003) Recoil-ion and electron momentum spectroscopy: reaction-microscopes. Reports on Progress in Physics, 66, 1463.

Willitsch, S. (2011) Experimental methods in cation spectroscopy, in Handbook of High-resolution Spectroscopy, Quack, M. and Merkt, F. (eds), John Wiley \& Sons, Ltd., Chichester, UK.

Witte, S., Zinkstok, R., Ubachs, W., Hogervorst, W., and Eikema, K. (2005) Deep-ultraviolet quantum interference metrology with ultrashort laser pulses. Science, 307, 400.

Wörner, H.J. and Merkt, F. (2011) Fundamentals of electronic spectroscopy, in Handbook of High-resolution Spectroscopy, Quack, M. and Merkt, F. (eds), John Wiley \& Sons, Ltd., Chichester, UK.

Young, L., Arms, D.A., Dufresne, E.M., Dunford, R., Ederer, D.L., Höhr, C., Kanter, E.P., Krässig, B., Landahl, E.C., Peterson, E.R. et al. (2006) X-Ray microprobe of orbital alignment in strong-field ionized atoms. Physical Review Letters, 97(83601), 083601.

Yudin, G.L. and Ivanov, M.Y. (2001) Nonadiabatic tunnel ionization: looking inside a laser cycle. Physical Review A, 64, 013409.

Yurchenko, S.N., Patchkovskii, S., Litvinyuk, I.V., Corkum, P.B., and Yudin, G.L. (2004) Laser-induced interference, focusing, and diffraction of rescattering molecular photoelectrons. Physical Review Letters, 93, 223003.

Zewail, A.H. (ed.) (1994) Femtochemistry - Ultrafast Dynamics of the Chemical Bond, World Scientific, Singapore.
Zholents, A.A. and Fawley, W.M. (2004) Proposal for intense attosecond radiation from an X-ray free-electron laser. Physical Review Letters, 92, 224801.

Zuo, T., Bandrauk, A.D., and Corkum, P.B. (1996) Laser-induced electron diffraction: a new tool for probing ultrafast molecular dynamics. Chemical Physics Letters, 259, 313.

\section{RELATED ARTICLES}

Eikema and Ubachs 2011: Precision Laser Spectroscopy in the Extreme Ultraviolet

Gallmann and Keller 2011: Femtosecond and Attosecond Light Sources and Techniques for Spectroscopy

Merkt and Quack 2011: Molecular Quantum Mechanics and Molecular Spectra, Molecular Symmetry, and Interaction of Matter with Radiation

Merkt et al. 2011: High-resolution Photoelectron Spectroscopy

Pratt 2011: High-resolution Valence-shell Photoionization

Quack 2011: Fundamental Symmetries and Symmetry Violations from High-resolution Spectroscopy

Wörner and Merkt 2011: Fundamentals of Electronic Spectroscopy

Willitsch 2011: Experimental Methods in Cation Spectroscopy 
